\title{
Ioffe-time distributions instead of parton momentum distributions in the description of deep inelastic scattering
}

\author{
V. Braun* \\ DESY, Notkestraße 85, D-22603 Hamburg, Germany \\ P. Górnicki \\ Center for Theoretical Physics and Institute of Physics, Polish Academy of Sciences, Al. Lotnikow 32/46, \\ PL-02-668 Warsaw, Poland \\ L. Mankiewicz \\ N. Copernicus Astronomical Center, Polish Academy of Science, ul. Bartycka 18, PL-00-716 Warsaw, Poland
}

(Received 19 October 1994)

\begin{abstract}
We argue that parton distributions in coordinate space provide a more natural object for nonperturbative methods compared to the usual momentum distributions in which the physics of different longitudinal distances is being mixed. To illustrate the advantages of the coordinate space formulation, we calculate the coordinate space distributions for valence quarks in the proton using the $\mathrm{QCD}$ sum rule approach. A remarkable agreement is found between the calculated and the experimentally measured $u$-quark distribution up to light-cone distances $\Delta^{-}=\Delta^{0}-\Delta^{3}$ of order $\sim 1 \mathrm{fm}$ in the proton rest frame. The calculation for valence $d$ quarks gives much worse results; the reasons for this discrepancy are discussed.
\end{abstract}

PACS number(s): 13.60.Hb, 11.55.Hx, 12.38.Lg, 14.20.Dh

\section{INTRODUCTION}

Deep inelastic lepton-hadron scattering has proved to be the best testing ground for perturbative QCD. Thanks to the celebrated factorization theorems [1], which can be derived quite rigorously in this case by using the operator product expansion (OPE), the entire $Q^{2}$ dependence of the cross section can be calculated perturbatively, while all dynamical effects of large distances are included in a set of one-particle parton distribution functions given at a certain reference scale. The determination of the set of partonic distributions, quark, antiquark, and gluon, is an ultimate goal for the experimental studies of the deep inelastic scattering, and also provides a challenging task for nonperturbative approaches to QCD.

In the past ten years remarkable progress has been made on the experimental side, and apart from the region of small Bjorken $x$, there is not much controversy regarding the existing parametrizations of parton distributions. The theoretical progress has been much more moderate. Apart from several quark-model or MIT bag model calculations, there have been relatively few attempts to determine parton distributions, e.g., from QCD sum rules. The problem has proved to be difficult for the theory. The purpose of this paper is to point out that a major part of theoretical problems in the calculations of parton distributions is due to the fact that distribution functions in momentum space for each particular value of the

\footnotetext{
${ }^{*}$ On leave of absence from St. Petersburg Nuclear Physics Institute, 188350 Gatchina, Russia.
}

momentum fraction $x$ receive contributions from both small and large longitudinal distances, which correspond to different physics and are difficult to treat simultaneously. We argue that longitudinal distance distributions are much easier from the theoretical point of view than momentum distributions. They can be extracted from data with marginal complications. In what follows we shall demonstrate that working with coordinate space distributions gives us a self-consistent formalism, which is not more complicated than the standard one and its relation to the OPE is much closer. We illustrate the advantages of this approach by the calculation of valence quark distributions in the framework of QCD sum rules.

Obviously, the longitudinal distance distributions are simply Fourier transforms of the momentum distributions. For example for the valence quark distribution one gets

$$
Q_{\mathrm{val}}\left(z, \mu^{2}\right)=\int_{0}^{1} d u \cos (u z) q_{V}\left(u, \mu^{2}\right)
$$

where $u$ is the momentum fraction. The physical interpretation of the variable $z$ has been discussed in the literature for a long time $[2,3]$. In the center-of-mass (c.m.) system of the target a deep-inelastic probe $\gamma^{*}$ (photon or neutrino) is converted into a quark-antiquark pair at some space-time location. At large $Q^{2}$ the pair travels with the speed of light along a lightlike path, interacts with the nucleon and is converted back into the probe. The time interval between the conversion points $\gamma^{*} \rightarrow \bar{q} q$ and $\bar{q} q \rightarrow \gamma^{*}$ in the c.m system, so called Ioffe time $\tau_{I}$, measures the lightlike distance essential for the process. The Lorentz-invariant variable related to $\tau_{I}$ is denoted by $z$. In the c.m. system the relation between these two 
variables takes a simple form $\tau_{I}=z / M$, where $M$ is the mass of the target (nucleon). For simplicity we shall call $z$ just the Ioffe time.

The idea to study $z$ distributions in order to understand the relative importance of various light-cone distances for deep inelastic scattering was proposed as early as 1970 [3]. There, the first analysis based on experimental data available at that time was presented. The experimental information about the Ioffe-time distributions available today is summarized in Fig. 1. We plot Fourier transforms for two representative sets of parametrizations [6], the next-to-leading order (NLO) Glück, Reya, Vogt parametrization [4] and the CTEQ parametrization [5], for the valence quark $(u$ and $d)$, quark plus antiquark, and gluon distributions at $Q^{2}=4 \mathrm{GeV}^{2}$. Exact definitions are given in Sec. II, where the connection of the Ioffe-time distributions and the OPE is discussed in detail. We note that all existing parametrizations are in fairly good agreement at small $z$, while at large $z$ there is some discrepancy. In order to get some insight about the transition from the momentum to the coordinate space, we plot in Fig. 2 the Ioffe-time valence quark distribution corresponding to a simple ansatz

$$
q_{V}(u)=N u^{\alpha}(1-u)^{\beta}
$$

with $\alpha=-0.5$ and $\beta=3$, as suggested by the Regge theory and the perturbative QCD arguments, respectively. The normalization factor $N$ ensures that $Q_{\mathrm{val}}(0)=$ $\int_{0}^{1} d u q_{V}(u)=1$. The shape of $Q_{\text {val }}$ depends very weakly on the exact value of the parameter $\beta$ as long as $\alpha \ll \beta$. The large- $z$ behavior can readily be obtained from the

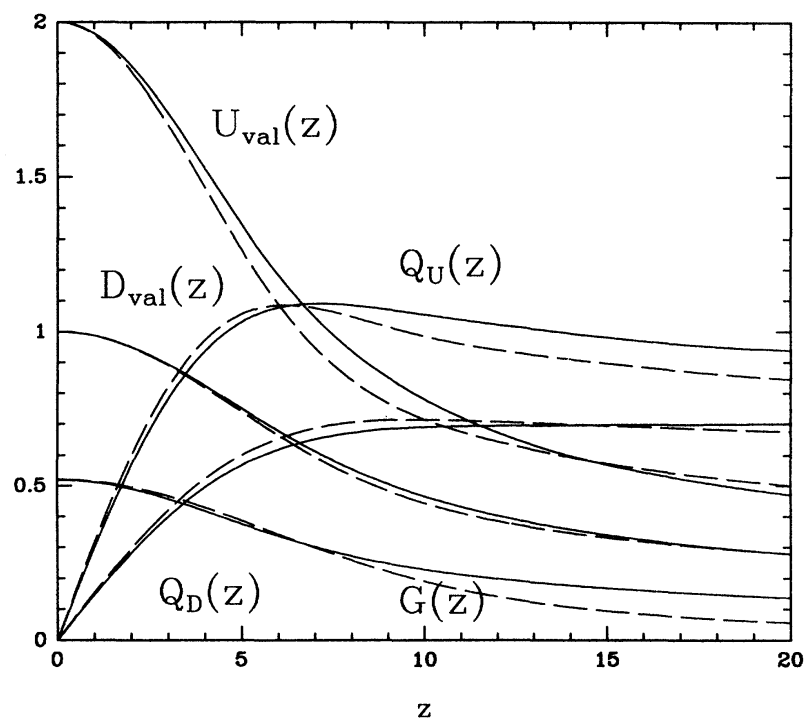

FIG. 1. Ioffe-time distributions for partons at the scale $\mu^{2}=4 \mathrm{GeV}^{2}$. The solid line denotes the Glück-Reya-Vogt (GRV) [4] set of parametrizations, the dashed line denotes CTEQ [5] parametrization. $U_{\mathrm{val}}(z)$ and $D_{\mathrm{val}}(z)$ are the valence quarks distributions, $Q_{U}(z)$ and $Q_{D}(z)$ are the $C$-even up and down quark distributions, and $G(z)$ is the gluon distribution.

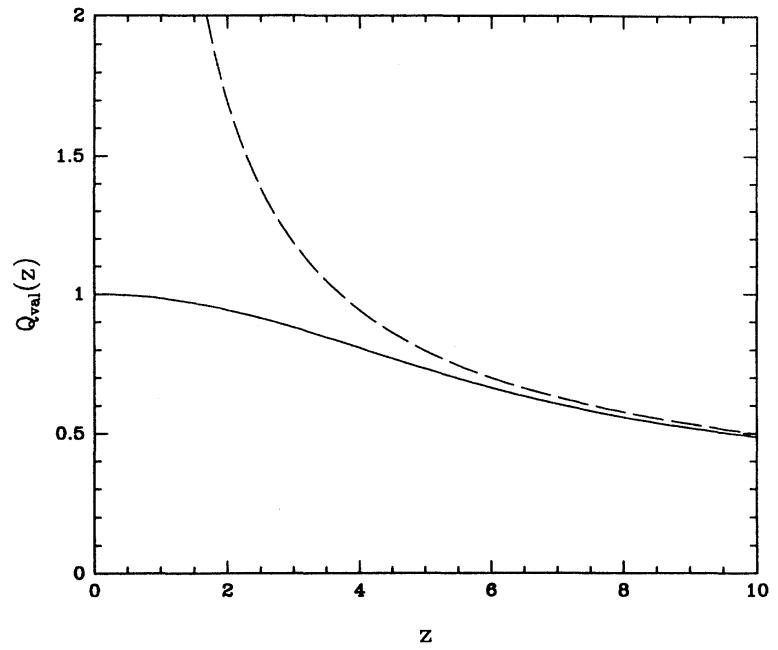

FIG. 2. Model of the Ioffe-time valence-type distribution corresponding to the simple ansatz $q_{V}(u)=N u^{\alpha}(1-u)^{\beta}$ with $\alpha=-0.5$ and $\beta=3$. The normalization is such that $\int_{0}^{1} d u q_{V}(u)=1$. The dashed line shows the asymptotic expansion (2). Note that it matches almost perfectly the true behavior for $z \geq 6$.

standard expressions for the asymptotic expansion of hypergeometric functions: ${ }^{1}$

$Q(z) \sim-\sin \left(\frac{\pi}{2} \alpha\right) \frac{\Gamma(\alpha+1)}{z^{\alpha+1}}+\beta \cos \left(\frac{\pi}{2} \alpha\right) \frac{\Gamma(\alpha+2)}{z^{\alpha+2}}+\cdots$.

As anticipated, the large- $z$ asymptotics of $Q(z) \sim z^{\alpha+1}$ is fixed by the Regge behavior. The valence quark distributions decrease at large $z$ as $\sim 1 / \sqrt{z}$. The sea quark and gluon distributions should approach a constant, or even may be rising functions of $z$ at large $z$.

An interesting question is at which values of $z$, or equivalently at which longitudinal distances, the behavior of parton distributions is already determined by the Regge asymptotics. The dashed line on Fig. 2 shows the asymptotic expansion of the function $Q_{\mathrm{val}}(z)$ which matches almost perfectly the true behavior when $z \geq 6$. Because in the target (lab) frame $z=\frac{1}{2} M \delta$, where $\delta$ denotes the distance along the lightcone, in the case of the nucleon these values of $z$ correspond to the light-cone distances $^{2} \delta \geq 2.5 \mathrm{fm}$. A nonperturbative calculation of the parton distribution in this region is an extremely intricate theoretical problem, which is essentially equivalent to providing a nonperturbative input to the Lipatov's Pomeron [7] (or Reggeon, in the case of valence distributions). On the other hand, calculation of the distributions at sufficiently small $z$ may be within reach of

\footnotetext{
${ }^{1}$ The asymptotical expansion contains in addition oscillating terms $\sim 1 / z^{\beta+1}$ but they can be neglected since $\alpha \ll \beta$ in realistic case.

${ }^{2}$ To avoid confusion, note that we are speaking here about light-cone distances, which in our definition are a factor 2 larger than physical longitudinal distances.
} 
existing models, the QCD sum rules, or the lattice calculations. Note that all parton distributions represented in Fig. 1 are very smooth at small $z$, which suggests that one should be able to reproduce them in this region with only few terms in the Taylor expansion around $z=0$. These terms are related to the first few moments of the momentum distributions, in other words to nucleon expectation values of a few local operators of low dimension (see below). The most important question is whether there exists a "matching window," where both the Regge asymptotic formulas and the small $z$ expansion are applicable. Provided the answer is positive, one could hope to get a quantitative description of the parton distributions in the whole $z$ range, matching these two different inputs at a certain intermediate value of $z$ (note similarity with the usual QCD sum rule program). Thus, the problem of calculating the parton distributions can be posed as a problem of calculating the distributions at distances of order 2-3 fm at the lightcone. As we shall see below, the standard QCD sum rules are sufficient for this purpose for the valence $u$-quark distributions, but fail for the $d$ quarks.

Playing around with typical parametrizations for parton distributions which are used in modern experimental analysis, one can convince oneself that in all cases the onset of the Regge behavior corresponds to values of $z \sim$ 5-8, see Fig. 1. Another useful example is given by the polarized gluon distribution. In this case we define

$$
\Delta G\left(z, \mu^{2}\right)=\int_{0}^{1} d u u \sin (u z) \Delta g\left(u, \mu^{2}\right),
$$

where $\Delta g\left(u, \mu^{2}\right)$ is the usual polarized gluon distribution depending on the momentum fraction $u$ (see Sec. II for details). Note that the gluon polarization

$$
\Delta g=\int_{0}^{1} d u \Delta g(u)=\int_{0}^{\infty} d z \Delta G(z) .
$$

A typical shape of $\Delta G(z)$ is shown in Fig. 3. It has been obtained with a simple model of $\Delta g(u)=N_{G} u^{\alpha}(1-u)^{\beta}$, and the solid curve corresponds to $\alpha=0$ and $\beta=4$. The normalization constant $N_{G}$ is chosen in such a way that the gluon polarization $\Delta g=0.5$. The two shortdashed curves were obtained by taking $\beta$ equal to 3.5 and 4.5, respectively, and keeping $\alpha=0$ and $\Delta g=0.5$ fixed. The corresponding variation of $\Delta G(z)$ is rather mild, and one can conclude that the behavior of $\Delta g(u)$ at small $u$ combined with the value of $\Delta g$ determine to a large extent the shape of $\Delta G(z)$. By the same argument, knowledge of $\Delta G(z)$ up to the point of maximum, which is again at $z \sim 6$, is enough to estimate the value of $\Delta g$ within, say, $50 \%$ accuracy. Note, however, that because of the more complicated shape of $\Delta G(z)$, asymptotic expansion shown by the long-dashed curve in Fig. 3 starts to be valid at larger values of $z \sim 10$.

Our presentation is organized as follows. In Sec. II we discuss the theoretical framework for the introduction of parton distributions in coordinate space as matrix elements of nonlocal operators, and we describe their $Q^{2}$ evolution. The presentation in this section mainly follows Refs. $[8,9]$. In Sec. III we give the QCD sum rule

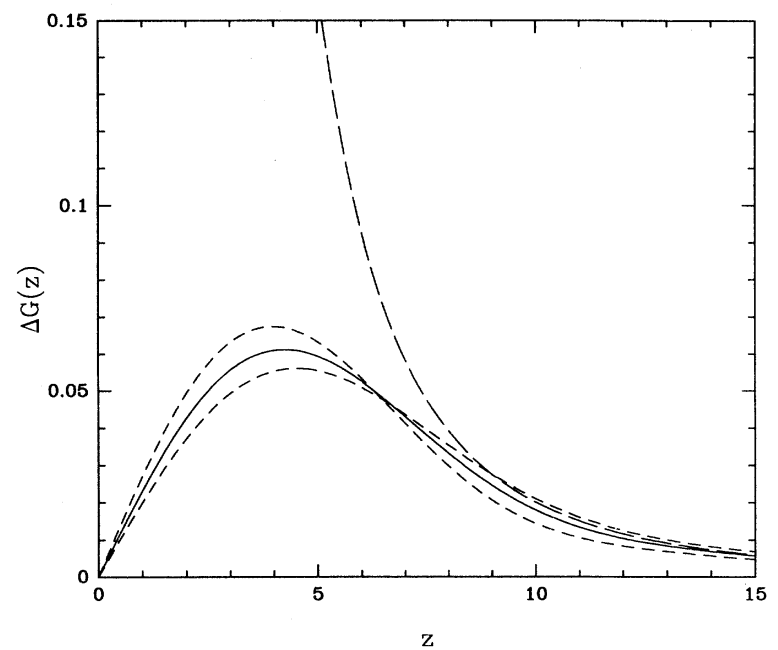

FIG. 3. Model of the Ioffe-time distributions for polarized gluon density $\Delta g(u)=N_{G} u^{\alpha}(1-u)^{\beta}$ with $\alpha=0$ and $\beta=4$. The normalization constant $N_{G}$ is chosen in such a way that the gluon polarization $\Delta g=0.5$. The two short-dashed curves were obtained by taking $\beta$ equal to 3.5 and 4.5 , respectively, and keeping $\alpha=0$ and $\Delta g=0.5$ fixed. The long-dashed curve is the asymptotic expansion (2).

calculation of valence quark distributions in coordinate space, and compare our approach to the direct calculation in the momentum space in Ref. [10]. Section 4 is reserved for a summary and conclusions. Some technical details of the sum rule calculation are presented in the Appendix.

\section{COVARIANT DEFINITION OF IOFFE-TIME DISTRIBUTIONS AND THEIR $Q^{2}$ EVOLUTION}

An intuitive discussion of the space-time picture of deep inelastic scattering in the late 1960 s can be put on a rigorous footing using the formalism of the operator product expansion (OPE). We are going to demonstrate that the Ioffe-time distributions arise naturally in this framework as reduced matrix elements of nonlocal string operators on the light cone. Our presentation essentially follows Refs. [8, 9].

It is well known that the deep-inelastic $e p$ scattering cross section is related to the matrix element:

$T_{\mu \nu}=\int d^{4} y \exp (i q y)\left\langle P\left|T\left[j_{\mu}(y)^{\dagger} j_{\nu}(0)\right]\right| P\right\rangle$,

where $|P\rangle$ represents a proton with momentum $P$ and $j$ is an electromagnetic current operator. This quantity describes the hadronic part of the process. The operator product expansion applied to $T[j(y) j(0)]$ gives rise to its systematic expansion in powers of the small parameter $\Lambda^{2} / Q^{2}$ where $\Lambda$ is the QCD scale of the order of 200 $\mathrm{MeV}$ and $Q^{2}=-q^{2}$ is the virtuality of the deep-inelastic probe. To the leading, twist-2 accuracy, i.e., when all powers of $\Lambda^{2} / Q^{2}$ are neglected, the quark and gluon op- 
erators appearing in the OPE of $T[j(y) j(0)]$ have the form

$\hat{\mathcal{O}}_{q}^{\mu_{1} \cdots \mu_{n}}(0)=\frac{1}{2} \bar{\Psi}(0)\left\{\gamma^{\mu_{1}} i D^{\mu_{2}} \cdots i D^{\mu_{n}}\right\}_{\mathrm{ST}} \Psi(0)$,

$\hat{\mathcal{O}}_{g}^{\mu_{1} \cdots \mu_{n}}(0)=\frac{1}{2}\left\{G^{\mu_{1} \nu}(0) i D^{\mu_{2}} \cdots i D^{\mu_{n-1}} G_{\nu}^{\mu_{n}}(0)\right\}_{\mathrm{ST}}$,

where $D^{\mu}=\partial^{\mu}-i g A^{\mu}$ denotes covariant derivative, and $\Psi$ and $G^{\mu \nu}$ are the quark field and the gluon field strength, respectively. The subscript ST denotes the symmetric and traceless part of a Lorentz tensor.

The operators defined in (6) form an irreducible representation of the Lorentz group. Their reduced matrix elements $\left\langle\left\langle O_{q}^{n}\right\rangle\right\rangle$ and $\left\langle\left\langle O_{g}^{n}\right\rangle\right\rangle$ are defined by

$$
\begin{aligned}
& \left\langle P\left|\hat{\mathcal{O}}_{q}^{\mu_{1} \cdots \mu_{n}}(0)\right| P\right\rangle=\left\langle\left\langle O_{q}^{n}\right\rangle\right\rangle\left\{P^{\mu_{1}} \cdots P^{\mu_{n}}\right\}_{\mathrm{ST}} \\
& \left\langle P\left|\hat{\mathcal{O}}_{g}^{\mu_{1} \cdots \mu_{n}}(0)\right| P\right\rangle=\left\langle\left\langle O_{g}^{n}\right\rangle\right\rangle\left\{P^{\mu_{1}} \ldots P^{\mu_{n}}\right\}_{\mathrm{ST}} .
\end{aligned}
$$

According to the standard analysis, the matrix elements are related to the moments of familiar quark and gluon distribution functions $q(x), \bar{q}(x)$, and $g(x)$ :

$$
\begin{aligned}
& \left\langle\left\langle O_{q}^{n}\right\rangle\right\rangle_{\mu^{2}}=\int_{0}^{1} d x x^{n-1}\left[q\left(x, \mu^{2}\right)+(-1)^{n} \bar{q}\left(x, \mu^{2}\right)\right], \\
& \left\langle\left\langle O_{g}^{n}\right\rangle\right\rangle_{\mu^{2}}=\int_{0}^{1} d x x^{n-1} g\left(x, \mu^{2}\right) .
\end{aligned}
$$

Equation (9) holds only for even values of $n$. Note that through renormalization the operators (6) acquire a scale dependence, which is related to the scale dependence of the parton distribution in (8) and (9).

An alternative representation for relations (9) and (8) has been noticed in [8] long ago, and we want to introduce it now. For that purpose let us define two lightlike vectors $n^{\mu}$ and $\Delta^{\mu}$, such that $n^{2}=\Delta^{2}=n \cdot \Delta=0$. Our convention is such that and for any vector $a, n \cdot a \equiv a^{+}=a^{0}+a^{3}$. The vector $\Delta$ is just proportional to $n, \Delta=\frac{1}{2} \delta n$, where $\delta=\Delta^{-}=\Delta^{0}-\Delta^{3}$ is the distance along the light cone. Following Ref. [8] we can write

$$
\langle P|\bar{\Psi}(\Delta)[\Delta ; 0] \Psi(0)| P\rangle_{\mu^{2}}=2(P \cdot n) \int_{0}^{1} d u\left[q\left(u, \mu^{2}\right) \exp (i u z)-\bar{q}\left(u, \mu^{2}\right) \exp (-i u z)\right]
$$

for quark distributions and

$$
\left\langle P\left|G_{\mu \xi}(\Delta)[\Delta ; 0] G_{\nu}^{\xi}(0)\right| P\right\rangle_{\mu^{2}} n^{\mu} n^{\nu}=4(P \cdot n)^{2} \int_{0}^{1} d u g\left(u, \mu^{2}\right) u \cos (u z)
$$

for gluons. In the above formulas $z=P \cdot \Delta$ and we have introduced the notation $[\Delta ; 0]$ for the path-ordered exponential,

$$
[\Delta ; 0]=\mathrm{P} \exp \left[i g \Delta_{\xi} \int_{0}^{1} d s A^{\xi}(\Delta s)\right]
$$

which is necessary for gauge independence of the parton distributions considered. An easy way to obtain the relations $(10),(11)$, and (13) is to insert the complete set of intermediate light-cone quark or gluon states between the field operators at the right-hand side (RHS) working in the Schwinger gauge: $\Delta \cdot A(\Delta)=0$. Taylor expansion in $\Delta$ of both sides of (10) and (11) gives exactly the set of relations (8) and (9) between the matrix elements of local operators and the moments of structure functions. Note that because $n$ and $\Delta$ are lightlike and proportional, the local operators arising here automatically are of twist 2 .

Fourier transformation of (10) and (11) gives a gauge-invariant definition of parton momentum distributions in terms of reduced matrix elements of leading twist nonlocal operators at the lightcone [8]. On the other hand, it is possible to demonstrate that the usual program of the OPE can be formulated directly in terms of nonlocal light-cone operators [9]. Thus, this formalism is consistent. For completeness, we quote the definition for the polarized gluon distribution $[11,12]$, see discussion after Eq. (3):

$$
\left\langle P, S\left|G_{\mu \xi}(\Delta)[\Delta ; 0] \tilde{G}_{\nu}^{\xi}(0)\right| P, S\right\rangle_{\mu^{2}} n^{\mu} n^{\nu}=4 i(P \cdot n)(S \cdot n) \int_{0}^{1} d u \Delta g\left(u, \mu^{2}\right) u \sin (u z),
$$

where $S$ is the nucleon spin vector normalized by $S^{2}=-M_{N}^{2}$.

Taking $C$-odd and $C$-even combinations of the left-hand side (LHS) of (10) we arrive at the definitions involving $C$-odd (valence) and $C$-even combinations of parton densities:

$$
\langle P|\bar{\Psi}(\Delta) \not h[\Delta ; 0] \Psi(0)| P\rangle_{\mu^{2}}+(\Delta \rightarrow-\Delta)=4(P \cdot n) \int_{0}^{1} d u q_{V}\left(u, \mu^{2}\right) \cos (z u)
$$

and

$$
\langle P|\bar{\Psi}(\Delta) \not h[\Delta ; 0] \Psi(0)| P\rangle_{\mu^{2}}-(\Delta \rightarrow-\Delta)=4 i(P \cdot n) \int_{0}^{1} d u\left[q\left(u, \mu^{2}\right)+\bar{q}\left(u, \mu^{2}\right)\right] \sin (z u),
$$

where $q_{V}\left(u, \mu^{2}\right)=q\left(u, \mu^{2}\right)-\bar{q}\left(u, \mu^{2}\right)$, and $\not h=n_{\mu} \gamma^{\mu}$.

As mentioned above, the conventional procedure is to Fourier transform the above formulas ending up with the parton distributions in momentum space. The main thrust of our paper is to point out that the matrix elements 
appearing on the LHS of Eqs. (10)-(15) have a clear physical interpretation as the parton distributions in the longitudinal coordinates, and are more adequate for the application of nonperturbative methods, retaining at the same time the whole physical content of the momentum space description. We define Ioffe-time distributions by

$$
\begin{aligned}
& \langle P|\bar{\Psi}(\Delta) \not h[\Delta ; 0] \Psi(0)| P\rangle_{\mu^{2}}+(\Delta \rightarrow-\Delta)=4(P \cdot n) Q_{\mathrm{val}}\left(z, \mu^{2}\right) \\
& \langle P|\bar{\Psi}(\Delta) \not h[\Delta ; 0] \Psi(0)| P\rangle_{\mu^{2}}-(\Delta \rightarrow-\Delta)=4 i(P \cdot n) Q\left(z, \mu^{2}\right)
\end{aligned}
$$

for quarks, and

$$
\begin{aligned}
& \left\langle P\left|G_{\mu \xi}(\Delta)[\Delta ; 0] G_{\nu}^{\xi}(0)\right| P\right\rangle_{\mu^{2}} n^{\mu} n^{\nu}=4(P \cdot n)^{2} G\left(z, \mu^{2}\right), \\
& \left\langle P, S\left|G_{\mu \xi}(\Delta)[\Delta ; 0] \tilde{G}_{\nu}^{\xi}(0)\right| P, S\right\rangle_{\mu^{2}} n^{\mu} n^{\nu}=4 i(P \cdot n)(S \cdot n) \Delta G\left(z, \mu^{2}\right),
\end{aligned}
$$

for nonpolarized and polarized gluon distributions, respectively. Comparing to (10)-(15) we arrive at the relations between the momentum and coordinate space distributions in (1), (3).

According to the standard discussion the scale dependence of the longitudinal momentum parton distributions is governed by the Gribov-Lipatov-Altarelli-Parisi (GLAP) equations written in momentum space. However, it is also possible to derive the corresponding evolution directly in coordinate space $[8,9]$. The coordinate-space version of the leading-log approximation (LLA) evolution equations has been obtained in [9] in the form of equations describing the normalization-point dependence of the nonlocal operators (10) and (11). Taking the forward nucleon matrix element and making use of relations (11), (14), and (15) one can derive evolution equations for Ioffe-time distributions $Q_{\text {val }}\left(z, \mu^{2}\right), Q\left(z, \mu^{2}\right)$, and $G\left(z, \mu^{2}\right)$.

To one-loop accuracy the scale dependence of the valence quark Ioffe-time distribution (16) is governed by

$$
Q_{\mathrm{val}}\left(z ; \mu_{2}^{2}\right)=Q_{\mathrm{val}}\left(z ; \mu_{1}^{2}\right)-\frac{\alpha_{S}}{2 \pi} C_{F} \ln \frac{\mu_{2}^{2}}{\mu_{1}^{2}} \int_{0}^{1} d u K(u) Q_{\mathrm{val}}\left(u z ; \mu_{1}^{2}\right)
$$

The kernel $K(u)$ is given by

$$
K(u)=\frac{1}{2} \delta(\bar{u})-\bar{u}-2\left[\frac{u}{\bar{u}}\right]_{+},
$$

where $\bar{u}=1-u$ and, for any function $f(u)$,

$$
\int_{0}^{1} d u\left[\frac{u}{\bar{u}}\right]_{+} f(u) \equiv \int_{0}^{1} d u \frac{u}{\bar{u}}[f(u)-f(1)] .
$$

In the flavor-singlet channel the evolution mixes, as expected, quark and gluon distributions $Q\left(z, \mu^{2}\right)(17)$ and $G\left(z, \mu^{2}\right)(18)$ :

$$
\left[\begin{array}{c}
Q\left(z ; \mu_{2}^{2}\right) \\
z G\left(z ; \mu_{2}^{2}\right)
\end{array}\right]=\left[\begin{array}{c}
Q\left(z ; \mu_{1}^{2}\right) \\
z G\left(z ; \mu_{1}^{2}\right)
\end{array}\right]-\frac{\alpha_{S}}{2 \pi} \ln \frac{\mu_{2}^{2}}{\mu_{1}^{2}} \int_{0}^{1} d u\left[\begin{array}{c}
C_{F} K_{Q Q}(u) N_{f} K_{Q G}(u) \\
C_{F} K_{G Q}(u) N_{C} K_{G G}(u)
\end{array}\right]\left[\begin{array}{c}
Q\left(u z ; \mu_{1}^{2}\right) \\
z G\left(u z ; \mu_{1}^{2}\right)
\end{array}\right],
$$

where

$$
\begin{aligned}
& K_{Q Q}(u)=\frac{1}{2} \delta(\bar{u})-\bar{u}-2\left[\frac{u}{\bar{u}}\right]_{+} \\
& K_{G G}(u)=\left(\frac{1}{6}+\frac{1}{3} \frac{N_{f}}{N_{C}}\right) \delta(\bar{u})-2\left[\frac{u}{\bar{u}}\right]_{+}+2\left(u^{3}-\bar{u}^{2}\right) \\
& K_{Q G}(u)=-\frac{1}{3} \bar{u}\left(2 \bar{u}^{2}+3 u\right) \\
& K_{G Q}(u)=-\delta(\bar{u})-2 \bar{u}
\end{aligned}
$$

where $N_{C}$ is the number of colors and $N_{f}$ is the number of active flavors. Equations (20) and (23) allow for a systematic study of the evolution of Ioffe-time parton densities exactly in the same manner as the conventional GLAP equations do for the longitudinal momentum parton densities. Indeed, let us rewrite (20) and (23) as the evolution equations for flavor nonsinglet and flavor singlet distributions:

$$
\mu^{2} \frac{\partial}{\partial \mu^{2}} Q_{\mathrm{val}}\left(z ; \mu^{2}\right)=-\frac{\alpha_{S}\left(\mu^{2}\right)}{2 \pi} C_{F} \int_{0}^{1} d u K(u) Q_{\mathrm{val}}\left(u z ; \mu^{2}\right)
$$

and 


$$
\mu^{2} \frac{\partial}{\partial \mu^{2}}\left[\begin{array}{c}
Q\left(z ; \mu^{2}\right) \\
z G\left(z ; \mu^{2}\right)
\end{array}\right]=-\frac{\alpha_{S}\left(\mu^{2}\right)}{2 \pi} \int_{0}^{1} d u\left[\begin{array}{l}
C_{F} K_{Q Q}(u) N_{f} K_{Q G}(u) \\
C_{F} K_{G Q}(u) N_{C} K_{G G}(u)
\end{array}\right]\left[\begin{array}{c}
Q\left(u z ; \mu^{2}\right) \\
z G\left(u z ; \mu^{2}\right)
\end{array}\right]
$$

which can be identified as the renormalization group equation (RGE) for twist-2 Ioffe-time distributions.

A beautiful feature of the RGE equations (25) and (26) is that they explicitly show the relevance of the short distance expansion: To calculate the QCD evolution of the distributions, one needs to know them at a certain reference scale at smaller values of the Ioffe time. Stated differently, the evolution equation for the nonlocal operators (10)-(13) involves these operators at quark-antiquark distances smaller than the initial separation. This is in contrast to the evolution of fragmentation functions, which is essentially nonlocal in the coordinate space [13].

As is well known, the integrodifferential equations (25) and (26) can be transformed into ordinary differential equations by Mellin transformation:

$$
F(z) \rightarrow \hat{F}(\nu)=\int_{0}^{\infty} d z z^{\nu-1} F(z)
$$

As a consequence we obtain, in the flavor nonsinglet case,

$$
\mu^{2} \frac{\partial}{\partial \mu^{2}} \hat{Q}_{\mathrm{val}}\left(\nu ; \mu^{2}\right)=-\frac{\alpha_{S}\left(\mu^{2}\right)}{4 \pi} \gamma(-\nu-1) \hat{Q}_{\mathrm{val}}\left(\nu ; \mu^{2}\right)
$$

where we have introduced the function

$$
\gamma(\nu)=2 C_{F} \int_{0}^{1} d u K(u) u^{\nu-1}
$$

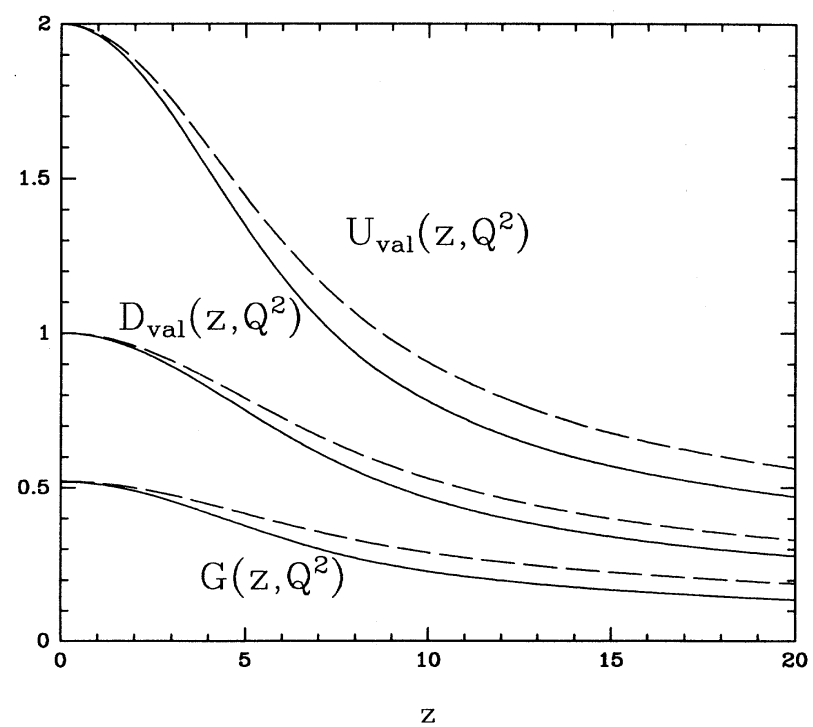

FIG. 4. Evolution of valence $U_{\text {val }}\left(z, Q^{2}\right)$ and $D_{\text {val }}\left(z, Q^{2}\right)$, and gluon $G\left(z, Q^{2}\right)$ Ioffe-time distributions. GRV [4] parametrization has been used. The solid line corresponds to $Q^{2}=4 \mathrm{GeV}^{2}$, the dashed line to $Q^{2}=20 \mathrm{GeV}^{2}$, respectively.
Equation (27) has the well-known solution

$$
\hat{Q}_{\mathrm{val}}\left(\nu ; \mu_{2}^{2}\right)=\left(\frac{\alpha_{S}\left(\mu_{2}^{2}\right)}{\alpha_{S}\left(\mu_{1}^{2}\right)}\right)^{\gamma(-\nu+1)) / b} \hat{Q}_{\mathrm{val}}\left(\nu ; \mu_{1}^{2}\right)
$$

where in QCD $b=\frac{11}{2}-\frac{1}{3} N_{f}$. The Ioffe-time distribution at the scale $\mu_{2}^{2}$ can be obtained with the help of the inverse Mellin transformation:

$$
\hat{F}(\nu) \rightarrow F(z)=\frac{1}{2 \pi i} \int_{c-i \infty}^{c+i \infty} d \nu z^{-\nu} F(\nu)
$$

Explicitly, one gets [9]

$$
\begin{aligned}
\hat{Q}_{\mathrm{val}}\left(z ; \mu_{2}^{2}\right)= & \int_{-\infty}^{\infty} \frac{d \nu}{2 \pi}\left(\frac{\alpha_{S}\left(\mu_{2}^{2}\right)}{\alpha_{S}\left(\mu_{1}^{2}\right)}\right)^{\gamma(1 / 2-i \nu) / b} \\
& \times \int_{0}^{\infty} d u u^{i \nu-1 / 2} \hat{Q}_{\mathrm{val}}\left(u z ; \mu_{1}^{2}\right) .
\end{aligned}
$$

Typical results of the low-scale evolution i.e., in the low $\mu^{2}$ range, are illustrated in Figs. 4 and 5. Figure 4 shows valence and gluon Gluck-Reya-Vogt (GRV) [4] Ioffe-time distributions evolved between $\mu^{2}=4$ and 20 $\mathrm{GeV}^{2}$. Figure 5 shows the same for $u$ - and $d$-quark distributions. Note that in this range of scales the evolution affects mainly the large- $z$ behavior of the distributions.

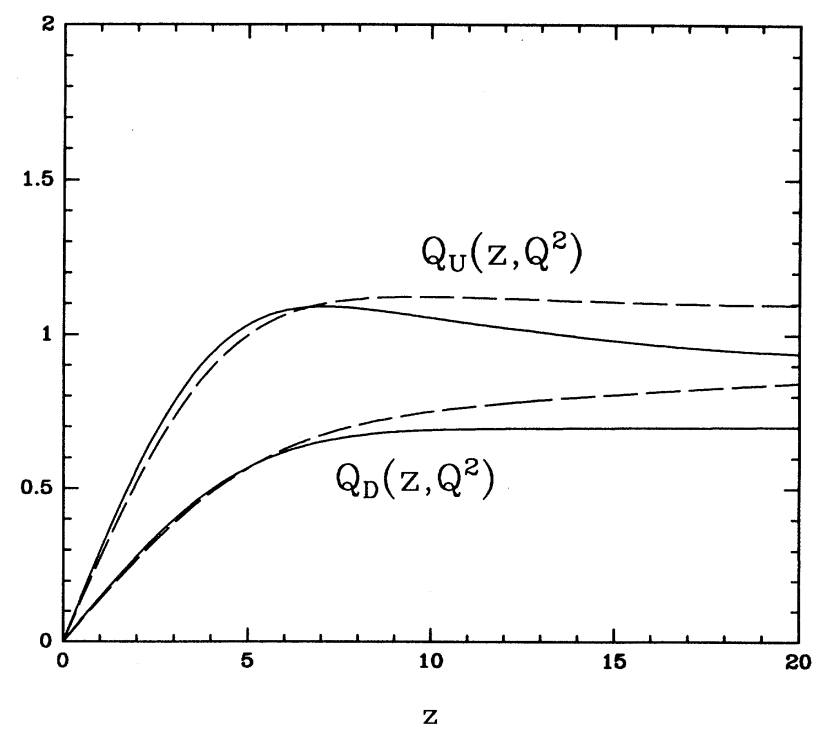

FIG. 5. Evolution of up and down quark $C$-even $Q_{U}\left(z, Q^{2}\right)$ and $Q_{D}\left(z, Q^{2}\right)$ Ioffe-time distributions. GRV [4] parametrization has been used. The solid line corresponds to $Q^{2}=4 \mathrm{GeV}^{2}$, the dashed line to $Q^{2}=20 \mathrm{GeV}^{2}$, respectively. 


\section{VALENCE QUARK DISTRIBUTIONS FROM QCD SUM RULES}

We can now summarize our discussion in the following way. We have analyzed the reduced matrix elements of QCD string operators of twist 2 as a function of the lightlike separation between fields. We have found very smooth behavior which makes such objects convenient for theoretical studies. It can be also shown that the large separations are dominated by the asymptotics of the corresponding structure function at small values of Bjorken $x$. Once this asymptotics is known from e.g., the Regge arguments, the remaining nontrivial information is contained in the domain of moderately large separations. This region could be accesible to presently developed analytical methods such as QCD sum rules, instanton models of QCD vacuum [14], or lattice calculations.

In this paper a QCD sum rule calculation is carried out for the valence quark distributions. In the last decade the QCD sum rule approach has been applied succesfully to a variety of problems, including estimation of hadron masses and couplings, elastic and transition form factors, etc. In the context of this paper it is necessary to mention the calculation of the fraction of proton momentum carried by gluons in Refs. $[15,16]$ and the calculation of structure functions at intermediate values of Bjorken variable in Ref. [10]. In what follows, we shall often refer to this latter analysis to compare the calculations in coordinate and momentum space.

The basic idea of the QCD sum rule technique is to use duality between hadronic and partonic representations of a suitable correlation function to extract the quantity of interest by requiring that the two descriptions match each other at intermediate scales. To calculate the valence quark distributions, we choose to work with

$$
\Pi^{u, d}=i^{2} \int d^{4} x d^{4} y \exp (i p \cdot x+i q \cdot y)\left\langle\Omega\left|T\left[\eta(x) \bar{\eta}(0) \hat{O}_{S}^{u, d}\left(y+\frac{\Delta}{2} ; y-\frac{\Delta}{2}\right)\right]\right| \Omega\right\rangle
$$

where

$$
\hat{O}_{S}^{i}\left(y+\frac{\Delta}{2} ; y-\frac{\Delta}{2}\right) \equiv \bar{\Psi}^{i}\left(y+\frac{\Delta}{2}\right) \gamma_{\mu} n^{\mu}\left[y+\frac{\Delta}{2} ; y-\frac{\Delta}{2}\right] \Psi^{i}\left(y-\frac{\Delta}{2}\right)+(\Delta \rightarrow-\Delta) .
$$

Here $i=u, d$ denotes quark flavor, $n^{\mu}=(1,0,0,-1)$ is the "unit" lightlike vector, $n^{2}=0$, and the splitting $\Delta^{\mu}$ is lightlike and proportional to $n^{\mu}$.

Finally, $\eta(x)$ is the standard interpolating current for the proton [17]

$$
\eta(x)=\epsilon^{a b c} u^{a}(x)^{T} C \gamma_{\mu} u^{b}(x) \gamma_{5} \gamma^{\mu} d^{c}(x),
$$

where $u(x)$ and $d(x)$ denote $u$ - and $d$-quark fields, respectively and $a, b, c$ are color indices.

According to Eq. (16) the proton matrix element of $\hat{O}^{i}\left(y+\frac{\Delta}{2} ; y-\frac{\Delta}{2}\right)$ defines the valence quark distribution. In the following we choose a special kinematics taking the momentum transfer $q_{\mu}$ to be lightlike $q^{2}=0$ and orthogonal to the interquark separation i.e., $\Delta \cdot q=0$. In this case, the nucleon contribution to the correlation function (31) can be extracted in the form

$$
\frac{1}{4} \operatorname{Tr} \not h \Pi^{u, d}=\frac{4 \lambda_{N}^{2}}{\left(p_{1}^{2}-M_{N}^{2}\right)\left(p_{2}^{2}-M_{N}^{2}\right)}(p \cdot n)^{2} \int_{0}^{1} d u q_{V}^{u, d}(u) \cos (u p \cdot \Delta)+\text { continuum , }
$$

where $p_{1}^{2}=p^{2}$ and $p_{2}^{2}=(p+q)^{2}$. The coupling $\lambda_{N}$ is defined by

$$
\langle\Omega|\eta(0)| P, N\rangle=\lambda_{N} u_{N}(P),
$$

where $u_{N}(P)$ is the nucleon spinor.

Note that the operator $\hat{O}$ in (32) is essentially the point-splitted vector current. In the limit $\Delta=0$ a Ward identity relates the three-point correlation function (31) to the derivative of the two-point correlation function of two nucleon currents

$$
\begin{aligned}
\Pi^{u, d}(\Delta=0) & =N^{u, v} \int_{0}^{1} d v n_{\mu} \frac{\partial}{\partial p_{\mu}} \Pi^{(2)}(p+v q) \\
\Pi^{(2)}(p) & =i \int d^{4} x \exp (i p \cdot x)\langle\Omega|T[\eta(x) \bar{\eta}(0)]| \Omega\rangle
\end{aligned}
$$

where $N^{u}=2, N^{d}=1$ are the numbers of valence quarks in the proton. The derivation of (35) and of a more general Ward identity for arbitrary separation $\Delta$ is given in the Appendix. Note that

$$
\frac{1}{4} \operatorname{Tr} \not n \Pi^{(2)}(p)=\frac{\lambda_{N}}{M_{N}^{2}-p^{2}}(p \cdot n)+\text { continuum. }
$$

Substituting (36) in (35) and comparing to (34) one obtains the normalization conditions

$$
\begin{gathered}
U_{\mathrm{val}}(0)=\int_{0}^{1} d u q_{u}(u)=N^{u}=2, \\
D_{\mathrm{val}}(0)=\int_{0}^{1} d u q_{d}(u)=N^{d}=1
\end{gathered}
$$

which are exact in the QCD sum rule approach, provided the Ward identity is not spoiled by the continuum subtraction (see below).

The main task is the calculation of the correlation function (31) in QCD. If both $p^{2}$ and $q^{2}$ are sufficiently large (and negative) the dominant contributions come 
from small distances $x$ and $y$ of order $1 /-p^{2}$ and $1 /-q^{2}$, respectively. Thus the standard machinery of the shortdistance expansion is applicable, allowing to express the result as a power series in terms of vacuum quark and gluon condensates. In the case of the forward matrix elements (i.e., for $q^{2}=0$ ) the situation is more involved because the relevant distances in the $t$ channel are not constrained by the external momenta and can be arbitrarily large.

The solution to this problem was first formulated by Balitsky [18]. The operator product expansion of the correlation functions of the type (31) has a twofold structure. Terms of the first type come from the region $x^{2} \sim$ $y^{2} \sim 1 /-p^{2}$ and are proportional to vacuum expectation values (VEV's) of local gauge invariant operators, multiplied by coefficient functions depending on $p_{1}^{2}, p_{2}^{2}$. In the following we refer to these terms as to local power corrections (LPC's).

Terms of the second type are called bilocal power corrections (BPC's) and correspond to the contributions of large $y^{2} \gg x^{2} \sim 1 / p^{2}$. In order to treat these terms for arbitrary $q$ in the "Bjorken limit" $p^{2} \sim q \cdot p \rightarrow \infty$ one should expand the $T$ product of nucleon currents,

$$
T[\eta(x) \eta(0)]=\sum_{n} C_{n}^{\mathrm{BL}}\left(x^{2}\right) \hat{S}^{n}(x ; 0),
$$

in a series of nonlocal, gauge-invariant "string" operators of increasing twist $n \hat{S}^{n}(x ; 0)$, cf. [9]. This expansion can be inserted into the correlation function (31), producing a power series in $1 / p^{2}$ with coefficients given by the correlation functions of two nonlocal light-cone operators

$$
\begin{array}{r}
i \int d y \exp (i q \cdot y)\langle\Omega| T\left[\hat{O}^{u, d}\left(y+\frac{\Delta}{2} ; y-\frac{\Delta}{2}\right)\right. \\
\left.\times \hat{S}^{n}(x ; 0)\right]|\Omega\rangle .
\end{array}
$$

In general, calculation of the correlator (39) requires construction of a specific sum rule and may be very complicated. Remarkably, we have found that the most important BPC's of dimension 6 can be evaluated exactly (i.e., related to the quark condensate) by using the equations of motion. The derivation essentially uses the Ward identity which we obtain in the Appendix.

It should be noted that the OPE for the correlation function (31) for $q^{2}=0$ is given by the sum of both LPC's and BPC's. In general only this sum has a physical meaning and is regularization scheme independent.

Apart from these general remarks, we shall not go into details of the calculation which is relatively straightforward. A few more remarks are necessary, however, concerning the specific techniques used in the QCD sum rules approach to suppress contributions of higher states and further taking them into account in the duality approximation.
To this end, the Borel transformation is applied to both sides of the sum rule, improving the convergence of the operator product expansion series and suppressing exponentially contributions of higher resonances. The rationale for keeping nonzero value of the momentum transfer $q$ in the above discussion is that in this kinematics one can consider $p_{1}^{2}=p^{2}$ and $p_{2}^{2}=(p+q)^{2}$ as independent variables and perform the Borel transformation in both momenta. The advantage of this procedure is that in the double dispersion relation

$$
\Pi=\int_{0}^{\infty} \int_{0}^{\infty} d s_{1} d s_{2} \frac{\rho\left(s_{1}, s_{2}\right)}{\left(s_{1}-p_{1}^{2}\right)\left(s_{2}-p_{2}^{2}\right)}+\cdots,
$$

where $\rho\left(s_{1}, s_{2}\right)$ denotes the spectral density, one can ignore subtraction terms and contributions corresponding to nondiagonal transitions with singularities in only one of the two variables. In the standard duality approximation higher resonances and the continuum contribution are taken into account by the following model for the physical spectral density:

$$
\begin{aligned}
\rho\left(s_{1}, s_{2}\right)= & \lambda_{N}^{2} \delta\left(s_{1}-M_{N}^{2}\right) \delta\left(s_{2}-M_{N}^{2}\right) \\
& \times\left\langle N, p_{1}\left|\hat{O}^{u, d}\right| p_{2}, N\right\rangle \\
& +\Theta\left(s_{1}-s_{0}\right) \Theta\left(s_{2}-s_{0}\right) \rho_{c}\left(s_{1}, s_{2}\right),
\end{aligned}
$$

where $\rho_{c}\left(s_{1}, s_{2}\right)$ is the corresponding spectral density calculated in perturbative QCD. Thus, by assumption, subtraction of the continuum contribution corresponds to constraining the integration region in $\left(s_{1}, s_{2}\right)$-plane to the duality region $s_{1}, s_{2}<s_{0}$.

In the theoretical part of the sum rule the double Borel transformation is performed using the general formula

$$
B\left\{\frac{\Gamma(\nu)}{\left[-\bar{v} p_{1}^{2}-v p_{2}^{2}\right]^{\nu}}\right\}=t^{2-\nu} \delta\left(v-\frac{M_{1}^{2}}{M_{1}^{2}+M_{2}^{2}}\right),
$$

where $t$ denotes the symmetric combination of Borel parameters $M_{1}^{2}$ and $M_{2}^{2}$ :

$$
t \equiv \frac{M_{1}^{2} M_{2}^{2}}{M_{1}^{2}+M_{2}^{2}}
$$

with $\bar{v}=1-v$. In the symmetric case $M_{1}^{2}=M_{2}^{2}=2 t$ the subtraction of the continuum contribution corresponds to the replacement

$$
\begin{aligned}
t^{n} \rightarrow & t^{n} E_{n}\left(t, s_{0}\right) \\
E_{n}\left(t, s_{0}\right)= & \left(1-e^{-s_{0} / t}\left[1+\frac{s_{0}}{t}+\cdots+\frac{1}{(n-1) !}\right.\right. \\
& \left.\left.\times\left(\frac{s_{0}}{t}\right)^{n-1}\right]\right) .
\end{aligned}
$$

in all terms of the OPE containing positive powers of the Borel parameter $t$.

An explicit calculation leads to the sum rule for the valence quark distributions:

$$
\begin{aligned}
e^{-M_{N}^{2} / t} \lambda_{N}^{2}\left\{\begin{array}{c}
U_{\mathrm{val}}(z) \\
D_{\mathrm{val}}(z)
\end{array}\right\}= & f_{\mathrm{pert}}^{u, d}\left(z, t, s_{0}\right)+f_{4}^{u, d}\left(z, t, s_{0}\right)\left\langle\left(\alpha_{s} / \pi\right) G^{2}\right\rangle+f_{6}^{u, d}(z, t)\langle\bar{q} q\rangle^{2} \\
& +f_{8}^{u, d}(z, t)\langle\bar{q} q\rangle \cdot\langle\bar{q} \sigma g G q\rangle+\cdots
\end{aligned}
$$


where we keep contributions of perturbation theory and operators up to dimension 8 . The coefficient functions are given by diagrams shown in Fig. 6. For the bilocal power corrections, examplified in Fig. 7 , we take into account all contributions related to contact terms, and neglect contributions of correlation functions involving explicitly the gluonic fields in addition to quark fields which arise from the first term in the Ward identity in (A4).

We find

$$
\begin{aligned}
& f_{0}^{u}(z)=\frac{1}{16 \pi^{4}} t^{3} E_{3}\left(t, s_{0}\right) \int_{0}^{1} d u\left(9 u \bar{u}^{2}+\bar{u}^{3}\right) \cos (u z) \\
& f_{0}^{d}(z)=\frac{1}{16 \pi^{4}} t^{3} E_{3}\left(t, s_{0}\right) \int_{0}^{1} d u\left(3 u \bar{u}^{2}+\bar{u}^{3}\right) \cos (u z) \\
& f_{4}^{u}(z)=\frac{1}{96 \pi^{3}} t E_{1}\left(t, s_{0}\right) \int_{0}^{1} d u\left[\left(4 \delta(u)+6 u-1+\frac{1}{3} z^{2} \bar{u}^{3}\right) \cos (u z)+z \bar{u}^{3} u^{-1} \sin (u z)\right], \\
& f_{4}^{d}(z)=\frac{1}{96 \pi^{3}} t E_{1}\left(t, s_{0}\right) \int_{0}^{1} d u\left[\left(2 \delta(u)+3 \bar{u}-u+\frac{1}{3} z^{2} \bar{u}^{3}\right) \cos (u z)+z \bar{u}^{3} u^{-1} \sin (u z)\right] \\
& f_{6}^{u}(z)=\frac{4}{3}, \\
& f_{6}^{d}(z)=\frac{2}{3} \cos z \\
& f_{8}^{u}(z)=-\frac{4}{9} t^{-1} \\
& f_{8}^{d}(z)=t^{-1}\left(-\frac{2}{9} \cos z+\frac{19}{54} z \sin z\right) .
\end{aligned}
$$

To keep the correct normalization of parton densities (37) the coupling $\lambda_{N}^{2}$ in (45) should be substituted by the corresponding sum rule [17],

$$
e^{-M_{N}^{2} / t} \lambda_{N}^{2}=\frac{1}{32 \pi^{4}} t^{3} E_{3}\left(t, s_{0}\right)+\frac{1}{32 \pi^{3}} t E_{1}\left(t, s_{0}\right)\left\langle\left(\alpha_{s} / \pi\right) G^{2}\right\rangle+\frac{2}{3}\langle\bar{q} q\rangle^{2}-\frac{2}{9} t^{-1}\langle\bar{q} q\rangle \cdot\langle\bar{q} \sigma g G q\rangle+\cdots,
$$

keeping the same terms in the OPE and using the same values of the Borel parameter $t$ and the continuum threshold $s_{0}$ as in (45). Note that the coefficients in front of the dimension 8 terms in (49) and (50) differ slightly from the corresponding ones in [10] and [17]. The reason is that the authors of $[10,17]$ evaluate the vacuum expectation values of nonlocal operators such as $\bar{\Psi}(0) \Psi(x) \bar{\Psi}(0) \Psi(x)$ reducing them to $\sim(\langle\bar{\Psi}(0) \Psi(x)\rangle)^{2}$ using the hypothesis of the dominance of the vacuum intermediate state, while we use this hypothesis in the calculation of local operators, such as $\bar{\Psi} D^{2} \Psi \bar{\Psi} \Psi$, only. This leads to extra terms as compared to [10,17]. These terms are, however, suppressed as $1 / N_{C}, N_{C}$ being the number of colors, and the difference is not important numerically.

Please note that all coefficient functions in the sum rule in (45) are smooth functions of the Ioffe time, in contrast with the sum rules for the momentum fraction distributions given in Ref. [10] which contain expansions involving singular functions. Indeed, making a Fourier transform of our expressions, we obtain the following sum rule for momentum fraction distributions of valence quarks:

$e^{-M_{N}^{2} / t} \lambda_{N}^{2} q_{V}^{u, d}(u)=\tilde{f}_{\mathrm{pert}}^{u, d}\left(u, t, s_{0}\right)+\tilde{f}_{4}^{u, d}\left(u, t, s_{0}\right)\left\langle\left(\alpha_{s} / \pi\right) G^{2}\right\rangle+\tilde{f}_{6}^{u, d}(u, t)\langle\bar{q} q\rangle^{2}+\tilde{f}_{8}^{u, d}(u, t)\langle\bar{q} q\rangle \cdot\langle\bar{q} \sigma g G q\rangle+\cdots$

with the coefficients

$$
\begin{aligned}
& \tilde{f}_{0}^{u}(u)=\frac{1}{16 \pi^{4}} t^{3} E_{3}\left(t, s_{0}\right)\left(9 u \bar{u}^{2}+\bar{u}^{3}\right) \\
& \tilde{f}_{0}^{d}(u)=\frac{1}{16 \pi^{4}} t^{3} E_{3}\left(t, s_{0}\right)\left(3 u \bar{u}^{2}+\bar{u}^{3}\right) \\
& \tilde{f}_{4}^{u}(u)=\frac{1}{96 \pi^{3}} t E_{1}\left(t, s_{0}\right)\left(3 \delta(u)+6 u-\left[\frac{1}{u^{2}}\right]_{+}\right) \\
& \tilde{f}_{4}^{d}(u)=\frac{1}{96 \pi^{3}} t E_{1}\left(t, s_{0}\right)\left(\delta(u)+4 \bar{u}-\left[\frac{1}{u^{2}}\right]_{+}\right) \\
& \tilde{f}_{6}^{u}(u)=\frac{4}{3} \delta(u) \\
& \tilde{f}_{6}^{d}(u)=\frac{2}{3} \delta(\bar{u}) \\
& \tilde{f}_{8}^{u}(u)=-\frac{4}{9} \delta(u) t^{-1} \\
& \tilde{f}_{8}^{d}(u)=-t^{-1}\left(\frac{2}{9} \delta(\bar{u})+\frac{19}{54} \delta^{\prime}(\bar{u})\right)
\end{aligned}
$$

where, for any test function $f(u)$,

$$
\int_{0}^{1} d u\left[\frac{1}{u^{2}}\right]_{+} f(u) \equiv \int_{0}^{1} d u \frac{1}{u^{2}}\left[f(u)-f(0)-u f^{\prime}(0)\right]
$$

It is easy to see that in high orders of the OPE the series of power corrections to the sum rules in coordinate and in momentum space will have the typical behavior

$$
\begin{aligned}
\frac{\langle A\rangle^{n}}{t^{n} n !} z^{n} & \rightarrow \frac{\langle A\rangle^{n}}{t^{n} n !} \delta^{(n)}(u), \\
\frac{\langle A\rangle^{n}}{t^{n} n !} z^{n} \cos (z) & \rightarrow \frac{\langle A\rangle^{n}}{t^{n} n !} \delta^{(n)}(\bar{u}),
\end{aligned}
$$

where $\langle A\rangle$ is the typical scale of vacuum fluctuations, of order (several hundred $\mathrm{MeV})^{2}$, and the $n$ ! suppression is due to the Borel transformation. For this reason the sum rules for Ioffe-time distributions can be justified theoretically at small values of $z$, while in the momentum space one faces a problem of the summation of the series 


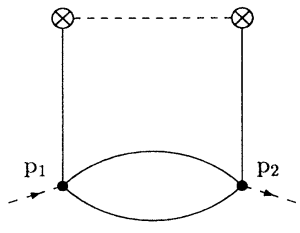

a

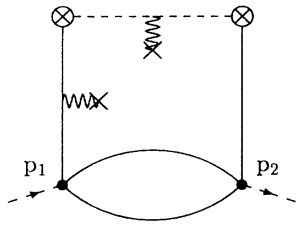

C

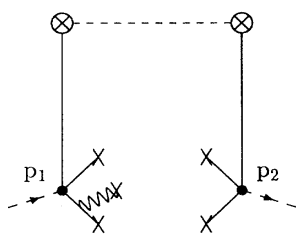

e

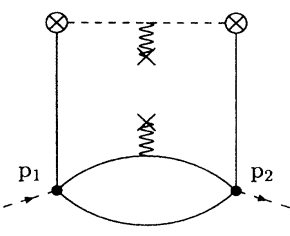

$\mathrm{b}$

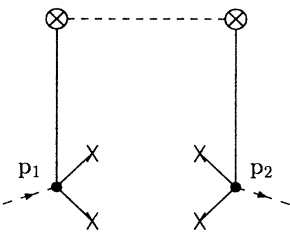

d

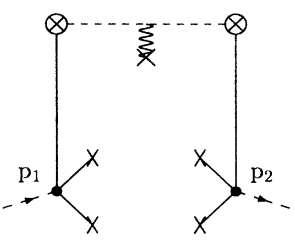

f
FIG. 6. Typical diagrams contributing to the OPE of the correlator (31).

containing singular functions.

Let us now elaborate on this point. The calculation of parton distributions in coordinate space is essentially on the same theoretical footing as the calculation of moments as matrix elements of local operators. Mathematically, information about the moments is coded in derivatives of the coordinate space distributions at $z=0$. Provided OPE converges fast enough, the $z$ distribution is well defined and can be calculated at sufficiently small $z$ by present nonperturbative approaches to QCD. In the particular technique of QCD sum rules, the results of calculations are usually assumed to be reliable provided contributions of vacuum condensates are sufficiently small, say stay within $30-40 \%$ of the total. It turns out that for valence quark distributions this criterium is satisfied for $z<3$. We shall see that in practice the sum rule for $u$-quark distributions works in a larger interval, and for $d$-quark distributions for a shorter interval; for other

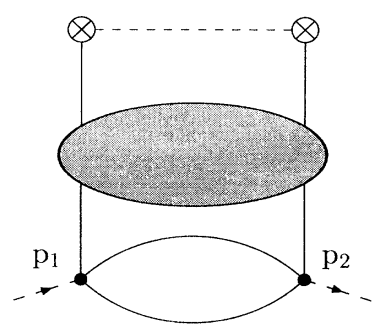

FIG. 7. Generic form of a bilocal correction in the OPE of the correlator (31). approaches, e.g., lattice calculations, the limitations can be different. Our point is that for sufficiently small $z$ one does not need to invoke any additional assumptions. The contributions of dimension 6 in (49) are respectable smooth functions at small $z$ and must be taken into account, independent of their bad behavior at large $z$ which produces $\delta$ functions after the Fourier transform.

In momentum space, the calculation of parton distributions pointlike in the Bjorken variable applies much more severe requirements to nonperturbative techniques, and in practice requires additional assumptions. In particular, the approach of Ref. [10] assumes that singular terms in the OPE do not affect calculation of parton distributions at intermediate values of momentum fraction $u$, and thus in this region all singular terms in the OPE can be neglected altogether (see also [19]). This would be true if summation of singular contributions produces a rather narrow smooth function with the support either in $u \sim 0$ or in $u \sim 1$ regions. Our task in this paper is not to criticize this particular assumption, but rather to make clear that assumptions of this kind are always necessary to deal with parton distributions in momentum space, and thus provide an additional input.

In fact, the assumption of Ref. [10] is nontrivial, and to our opinion requires a better justification than given there. Mathematically, the statement about calculability of coordinate-space distributions at sufficiently small $z$ does not imply calculability of momentum-space distributions at intermediate values of $u$. We find the neglect of singular contributions disturbing, since they are $100 \%$ essential for calculation of the moments, see Refs. $[15,16]$. Since the calculation of the parton distributions along the lines of Ref. [10] (and this paper) is only justified as the analytic continuation from the corresponding calculation of the moments [9], it is difficult for us to imagine that important contributions to the moments of the structure function will not show up in the distribution itself. Physically, the assumption about the small smearing of singular contributions implies existence of a certain second scale in the hadrons, affecting the momentum distributions. We feel that a further study of this question is necessary, to prove that smearing of singular contributions does not affect the whole region of the Bjorken variable. Again, we repeat that the advantage of coordinate space formulation is that it avoids making any assumptions of this kind, since singular contributions to not appear.

Let us proceed to the description of our results. In the numerical analysis we use standard values of the parameters accepted in the QCD sum rules for the nucleon, i.e., $t \sim 1 \mathrm{GeV}^{2}$ and $s_{0}=(1.5 \mathrm{GeV})^{2}$, and the following values for the condensates (the normalization point $1 \mathrm{GeV}$ is implied):

$$
\begin{aligned}
\langle\bar{q} q\rangle & =(250 \mathrm{MeV})^{3}, \\
\left\langle\left(\alpha_{s} / \pi\right) G^{2}\right\rangle & =0.012 \mathrm{GeV}^{4} \\
\langle\bar{q} \sigma g G q\rangle & =m_{0}^{2}\langle\bar{q} q\rangle ; \quad m_{0}^{2}=0.64 \mathrm{GeV}^{2},
\end{aligned}
$$

which correspond to the standard ITEP values rescaled to the normalization point $\mu^{2} \sim M_{N}^{2} \sim 1 \mathrm{GeV}^{2}$.

The QCD sum rule prediction for the valence $u$-quark Ioffe-time distribution in the proton $U_{\mathrm{val}}\left(z, \mu^{2} \sim 1 \mathrm{GeV}^{2}\right)$ 


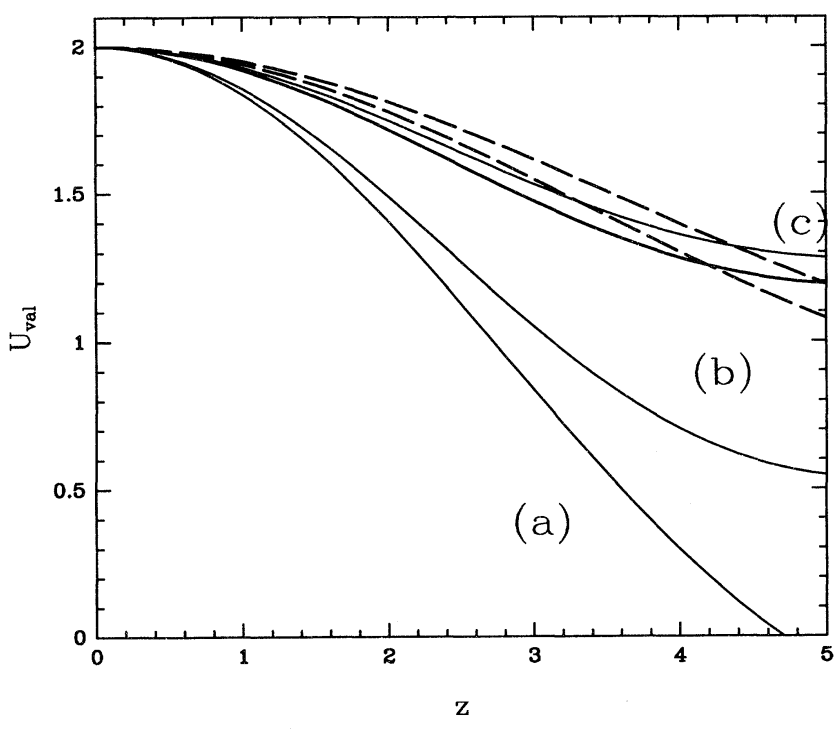

FIG. 8. QCD sum rules calculation of the valence $u$-quark Ioffe-time distribution function $U_{\text {val }}\left(z, \mu^{2}\right)$ at $\mu^{2} \sim 1 \mathrm{GeV}^{2}$. The thick solid line results from the OPE with operators with dimension $0,4,6$, and 8 . Thick dashed lines correspond to the leading-order QCD analysis of Ref.[20] at the scales $\mu^{2}=0.5$ and $1 \mathrm{GeV}^{2}$, respectively. The solid line marked (a) is the perturbative contribution to the sum rule. Lines (b) and (c) describe, respectively, the sum rules with operators of dimension 4 and 6 taken into account. Note that VEV of dimension 8 already gives a small contribution.

is shown as the thick solid line in Fig. 8 and compared with an "experimental" distribution. The latter has been obtained from the leading-order parametrization of Gluck, Reya, and Vogt [20] normalized at 0.5 $\mathrm{GeV}^{2} \leq \mu^{2} \leq 1 \mathrm{GeV}^{2}$. We find remarkable agreement up to rather large values $z \leq 4$, corresponding to longitudinal distances in the proton rest frame of nearly 2 $\mathrm{fm}$. When this QCD sum rule result is augmented by the assumption that $U_{\mathrm{val}}(z)$ is a sufficiently smooth function, and combined with large- $z$ behavior implied by the Regge theory [see Eq. (2)] it allows for complete reconstruction of $U_{\mathrm{val}}(z)$ and therefore of the distribution function. Lines marked as (a), (b), and (c) illustrate the relative importance of different contributions to the sum rule, and are obtained keeping in (45), (50) the perturbative terms only (a), adding the gluon condensate contribution (b), and adding in addition also the $\langle\bar{q} q\rangle^{2}$ terms (c). Note that the $U_{\text {val }}(z)$ distribution decreases at large $z$ more slowly than the perturbative prediction, which is mainly due to bilocal corrections arising from large distances in the $t$ channel. The latter can be calculated as contact terms. We remind that these terms are discarded altogether in the approach of Ref. [10].

Figure 9 shows stability of our prediction when the Borel parameter $t$ is varied between $1 \mathrm{GeV}^{2}$ (upper curve) and $1.5 \mathrm{GeV}^{2}$ (lower curve). One may conclude that in the region $z \leq 4$ the valence $u$-quark sum rule converges very fast and it is numerically stable.

A sum rule similar to the one given by Eq. (45) can be written for a nonzero value of the momentum transfer $q^{2}$

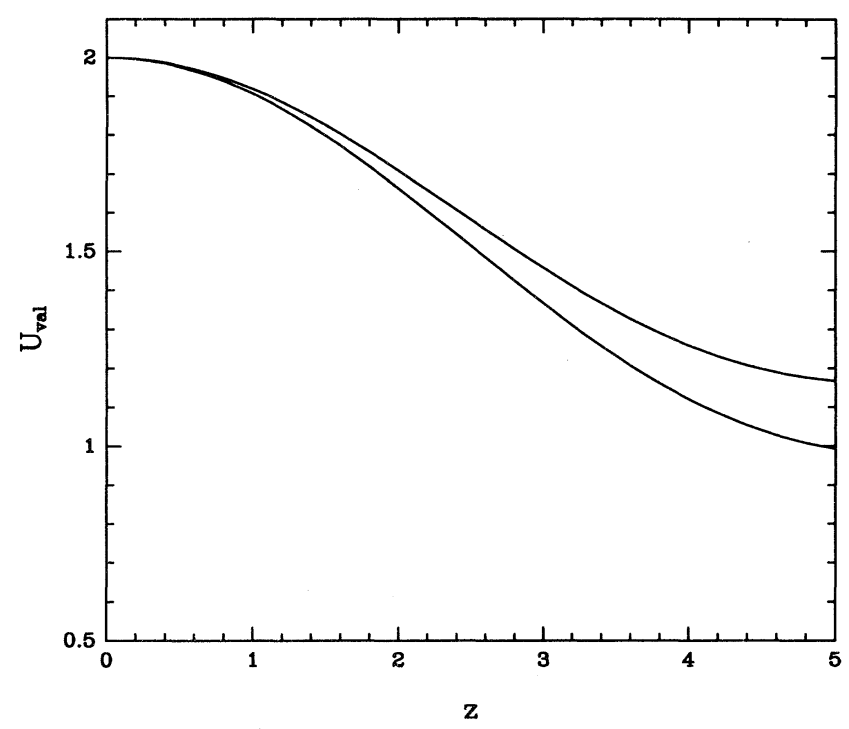

FIG. 9. Stability of the sum rule for $U_{\text {val }}(z)$ against variation of the Borel parameter $t$. The upper curve corresponds to $t=1 \mathrm{GeV}^{2}$, the lower to $t=1.5 \mathrm{GeV}^{2}$.

in the $t$ channel, allowing to study the radius of the valence quark distributions (cf. [21]). We have checked that the radius of the valence $u$-quark distribution obtained from the sum rule in (45) is close to the measured electromagnetic radius of the proton, which is encouraging.

The situation is not so good, unfortunately, for the valence $d$-quark distribution, see Fig. 10. The sum rule

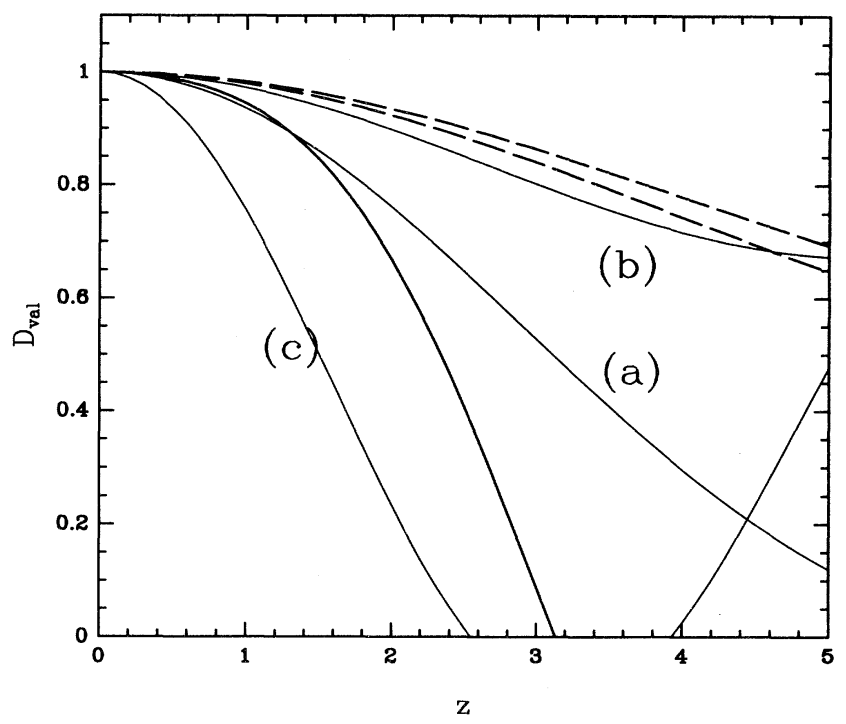

FIG. 10. QCD sum rules calculation of the valence $d$ quark Ioffe-time distribution function $D_{\text {val }}\left(z, \mu^{2}\right)$ at $\mu^{2} \sim 1$ $\mathrm{GeV}^{2}$. The thick solid line results from the OPE with operators of dimension $0,4,6$, and 8 . Thick dashed lines correspond to the leading-order QCD analysis of Ref. [20] at the scales $\mu^{2}=0.5$ and $1 \mathrm{GeV}^{2}$, respectively. The solid line marked (a) is the perturbative contribution to the sum rule. Lines (b) and (c) describe, respectively, the sum rules with condensates of dimension 4 and 6 taken into account. 
prediction for $D_{\text {val }}(z)$, shown as the thick solid line, is rather far from the leading-order GRV [20] parametrization, and is much more short range. As in Fig. 8, we also show contributions of various terms in the OPE to the final result. It is seen that taking into account the gluon condensate contribution (b) improves the prediction considerably compared to the perturbative result (a), but this tendency is destroyed by the contributions of dimension 6 (c) and dimension 8. In the case of the $d$-quark distribution the bilocal corrections corresponding to the contact terms are absent, and the problem arises because of the diagram in which all nucleon momentum is carried by a single quark, see Fig. 6 (d), which contributes a term $\sim \cos (z)$ (49). In the momentum space this contribution is proportional to $\delta(1-u)$, where $u$ is a fraction of longitudinal momentum carried by the quark, see (55). Another reason is the absence of the bilocal power correction of dimension 6 , contributing a term $\sim \delta(u)$ in (55), suggesting that terms of higher dimension $\sim \delta(u), \delta^{\prime}(u), \ldots$ can be important.

It turns out that the $d$-quark distribution is very sensitive to the numerical values of condensates of dimension 6 and 8 because of strong cancellation between corresponding terms. If we took $\bar{q} q=-(240 \mathrm{MeV})^{3}$ and $m_{0}^{2}=0.8 \mathrm{GeV}^{2}$, which correspond to the normalization point $\mu^{2}=0.5 \mathrm{GeV}^{2}$, we would obtain perfect agreement with the experimental analysis up to values of $z$ of order of 1 , where the sum rules prediction would abruptly turn down. Such a strong normalization-point dependence makes our prediction for $d$ quarks less reliable.

A favorable structure of the OPE for the $u$-quark distribution and the complications for $d$ quarks have, presumably, no physical relevance, and are due to the particular structure of the interpolating current (33). This choice is standard, but, as it follows from our analysis, not very convenient for the study of the $d$-quark distributions, since it implies that the correct behavior of this distribution is due to higher order power corrections.

A more detailed analysis of this problem goes beyond the scope of this paper. One could try a different interpolating current to improve the results for the $d$-quark distributions, or calculate radiative corrections to the sum rule, which generally tend to soften the parton distributions (i.e., make them more extended in the Ioffe time) and are expected to be especially important for $d$-quarks, see $[10]$.

However, it is worthwhile to demonstrate, at least semiquantitatively, that the higher order contributions indeed tend to smoothen the $\sim \delta(1-u)$ contribution of dimension 6 in the $d$-quark sum rule, and are potentially able to bring it to the agreement with the data. To this end we use the concept of nonlocal condensates, introduced in [22], which allows us to consider the effects of the final correlation length in the QCD vacuum, the property that is missing in the local operator product expansion.

Note that the contribution of the diagram in Fig. 6 (d) is essentially proportional to the vacuum expectation value of the nonlocal operator $\left[u^{a T}(x) C \gamma_{\xi} u^{b}(x)\right]\left[\bar{u}^{a}(0) C \gamma_{\xi} \bar{u}^{b T}(0)\right]$ which produces the expansion

$$
\begin{aligned}
\left\langle\left[u^{a T}(x) C \gamma_{\xi} u^{b}(x)\right]\left[\bar{u}^{a}(0) C \gamma_{\xi} \bar{u}^{b T}(0)\right]\right\rangle & \\
& =-\frac{2}{3}\langle\bar{q} q\rangle^{2}\left[1+\frac{1}{8} m_{0}^{2} x^{2}+\cdots\right],
\end{aligned}
$$

where we have assumed the factorization to evaluate the coefficients. It is the expansion into the sum of local operators that generates the series of power corrections proportional to derivatives of $\delta(1-u)$ in the sum rule for the momentum fraction distributions. As noted in [22], this expansion misses an important property of the correlation functions in Euclidian space, which is in existence of the final correlation length in the physical vacuum. To illustrate this point, let us consider the exponential parametrization

$$
\begin{aligned}
\left\langle\left[u^{a T}(x) C \gamma_{\xi} u^{b}(x)\right]\right. & {\left.\left[\bar{u}^{a}(0) C \gamma_{\xi} \bar{u}^{b T}(0)\right]\right\rangle } \\
= & -\frac{2}{3}\langle\bar{q} q\rangle^{2} \int_{0}^{\infty} d \nu e^{\left(x^{2} / 4\right) \nu} f(\nu) .
\end{aligned}
$$

Moments of the function $f(\nu)$ are determined by vacuum expectation values of local operators. The first few of them in the factorization approximation are fixed to be

$$
\begin{aligned}
\int_{0}^{\infty} d \nu f(\nu) & =1 \\
\int_{0}^{\infty} d \nu \nu f(\nu) & =\frac{1}{2} \frac{\langle\bar{q} g \sigma G q\rangle}{\langle\bar{q} q\rangle} \equiv \frac{1}{2} m_{0}^{2} .
\end{aligned}
$$

On the other hand, one generally expects that the correlation functions in QCD decrease exponentially in Euclidian space, suggesting that

$\left\langle\left[u^{a T}(x) C \gamma_{\xi} u^{b}(x)\right]\left[\bar{u}^{a}(0) C \gamma_{\xi} \bar{u}^{b T}(0)\right]\right\rangle \sim \exp \left[-M_{D} \sqrt{-x^{2}}\right]$

at large $x^{2} \rightarrow-\infty$, where $M_{D}$ is the correlation length which, loosely speaking, may be associated with the diquark mass. It is easy to see that this behavior corresponds to the asymptotics

$$
f(\nu) \sim e^{-M_{D}^{2} / \nu}
$$

at small $\nu$. Note that the expansion into the sum of local operators corresponds to the expansion of $f(\nu)$ in $\delta$ functions at $\nu=0$.

The effect of using the nonlocal condensate in the sum rules is easy to evaluate. In coordinate space, insertion of (60) amounts to the substitution of the coefficient function $f_{6}^{d}$ in $(49)$ by

$$
f_{\mathrm{NLC}}^{d}(z, t)=\frac{2}{3}\langle\bar{q} q\rangle^{2} t \int_{0}^{1} d u u f(\bar{u} t) \cos (u z) .
$$

In momentum space this replacement is simply

$$
\tilde{f}_{6}^{d}(u)=\frac{2}{3} \delta(\bar{u}) \rightarrow \tilde{f}_{N L C}^{d}(u, t)=\frac{2}{3} t u f(\bar{u} t) .
$$

For numerical estimates we choose a simple model

$$
f(\nu)=\frac{\left(M_{D}^{2}\right)^{a-2}}{\Gamma(a-2)} \nu^{1-a} e^{-M_{D}^{2} / \nu}
$$

with two parameters $M_{D}$ and $a$. Equation (61) leads to the constraint

$$
a-3=2 \frac{M_{D}^{2}}{m_{0}^{2}}
$$

so that we only need to specify the correlation length. Its 
value has a direct physical meaning and is related to the difference between masses of heavy baryons, containing $b$ quark and the $u u$ pair, and the mass of the $b$ quark. $^{3}$

In Fig. 11 we show the right-hand side (RHS) of (65) as a function of the momentum fraction $u$ for the Borel parameter $t=1 \mathrm{GeV}^{2}$ and for two choices of $M_{D}=700$ $\mathrm{MeV}$ and $1 \mathrm{GeV}$. We see that the main effect of the finite correlation length is to push nonperturbative effects $\sim\langle\bar{q} q\rangle^{2}$ away from the region $u \sim 1$. This may seem contrary to the physical intuition, but not necessarily so, because the effects of the quark condensate are qualitatively similar to the introduction of the constituent mass. We remark that this picture contradicts the expectations of Ref. [10]: Summation of singular contributions not only fails to produce a narrow function with a support concentrated at $u \rightarrow 1$, but, on the contrary, the nonperturbative contributions die away at $u \rightarrow 1$ faster than any power of $1-u$. We stress that this is a direct consequence of the final correlation length in the $Q C D$ vacuum. Our model estimates presented in Fig. 11 show that the resulting contributions are important at least up to $u \sim 0.6$.

Numerical results for the valence $d$ quark distribution $D_{v}(z)$ with the nonlocal condensate are shown in Fig. 12.

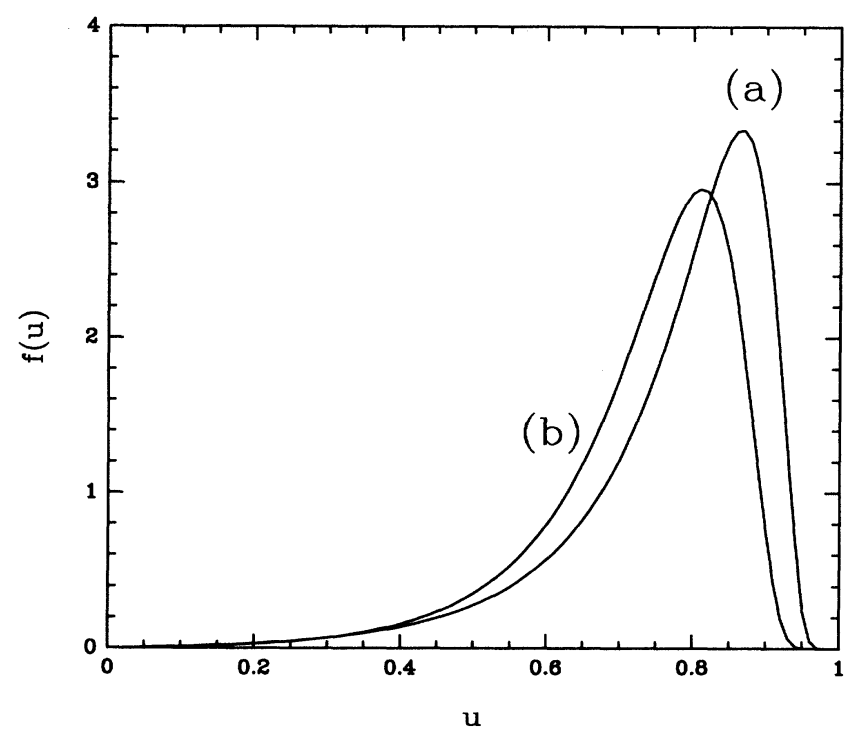

FIG. 11. The nonlocal condensate contribution to the $d$ quark sum rule, see Eq. (65). Lines marked as (a) and (b) correspond to the diquark mass parameters $M_{D}=\mathrm{GeV}$ and $1.0 \mathrm{GeV}$, respectively. Borel parameter $t=1 \mathrm{GeV}^{2}$.

\footnotetext{
${ }^{3}$ We do not discuss this issue in detail, referring to a wellknown relation between the asymptotics of the quark propagator at large distances in Euclidian space, and the difference between heavy meson and quark masses in the heavy quark limit [23]. This difference is usually denoted by $\bar{\Lambda}$ and is one of the main observables in the heavy quark effective theory. For baryons the situation is quite similar. The range of values of $M_{D}$ used here corresponds to the estimates found in the literature.
}

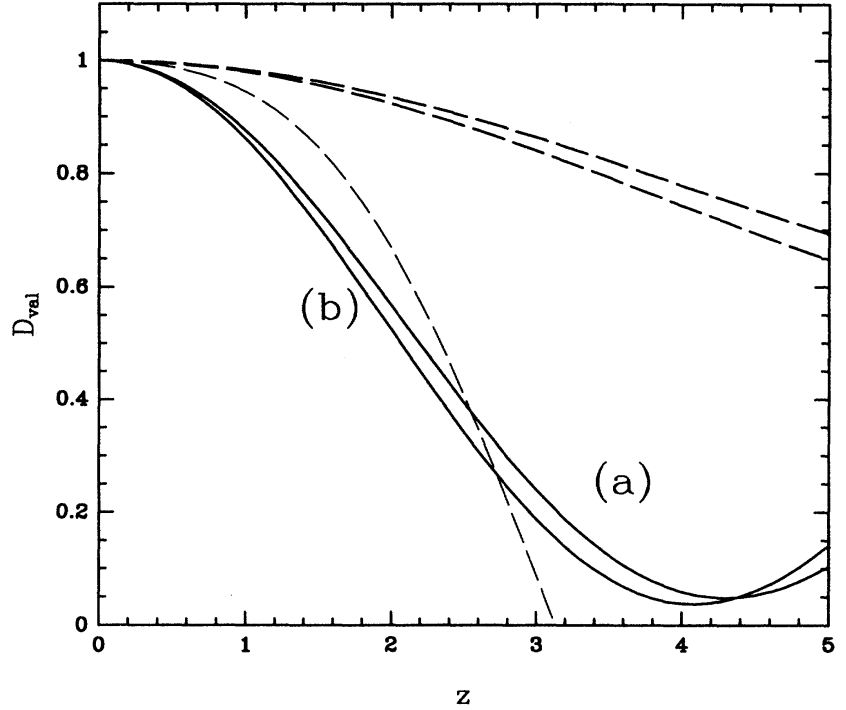

FIG. 12. QCD sum rules calculation of the valence $d$ quark Ioffe-time distribution function $D_{\text {val }}(z)$ (solid lines). VEV of dimension 6 and 8 has been replaced by the phenomenological model of nonlocal four-quark condensate, Eq. (60). Labels (a) and (b) refer to the diquark mass parameters $M_{D}=0.7$ and $1.0 \mathrm{GeV}$, respectively. Thick dashed lines correspond to the leading-order QCD analysis of Ref. [20] normalized at $\mu^{2}=0.5$ and $1 \mathrm{GeV}^{2}$, respectively. The dashed line shows results of the standard sum rule (45).

We see that the situation improved somewhat at $z>2$, although this type of contributions alone is not able to restore the agreement to the data. A better agreement can be obtained by choosing a larger value of the mixed condensate parameter $m_{0}^{2}$, but this possibility is not very attractive. We expect, however, that the results will be substantially improved by taking into account radiative corrections. An inspection shows that the sum rule for $d$ quark distributions can also be saved by a large bilocal power correction, contributing a term $\sim z^{2}$ in coordinate space (alias $\sim \delta^{\prime \prime}(u)$ in momentum space). These corrections are difficult to evaluate, however, and we do not attempt this task in the present paper. An experience of QCD sum rule calculations generally suggests that if there are indications that the sum rule is affected by contributions of high order in the OPE, it is advisable to use different interpolating currents for the participating hadrons.

\section{SUMMARY AND CONCLUSIONS}

We suggest to use Ioffe-time distributions, the distributions of invariant longitudinal distances $z$ essential in a deep-inelastic scattering process, as a suitable alternative to the conventional description in terms of momentum fraction parton distributions. The advantage of this formulation is that contributions of large and small longitudinal distances that correspond in fact to different physics, become in this approach separated. The large distances of distributions in coordinate space are governed by the Regge theory, and can be taken as an in- 
put, while the calculation at moderate distances can be within reach of current nonperturbative approaches, e.g., the lattice QCD. We illustrate these advantages using the QCD sum rule technique to calculate valence quark distributions, and compare it to the corresponding calculations in the momentum space. Although the sum rules derived in this paper are rather preliminary and can be improved significantly by taking into account further corrections, we obtain a very good description of the $u$ quark distributions. The results for the $d$ quark distributions are worse, the reason is an unfavorable structure of the operator product expansion series in this case, where higher order terms are important.

We believe that our results can be improved by making a state-of-the-art QCD sum rule analysis, or with different techniques. In particular, we expect that Ioffe-time distributions may be feasible for lattice calculations and for instanton models of the QCD vacuum of the type suggested in Ref. [14]. It would be most interesting to constrain in this way the polarized gluon distribution which is poorly known.

\section{ACKNOWLEDGMENTS}

This work has been supported by KBN Grant No. 2 P302 14306.

\section{APPENDIX}

In this Appendix we derive a Ward identity for the correlation function (31) useful in practical calculations.
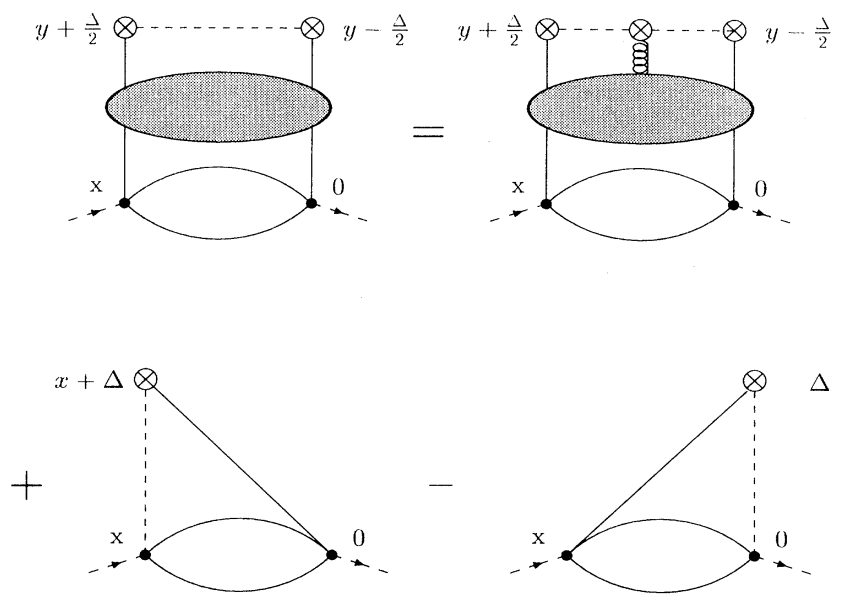

FIG. 13. Graphical illustration of the Ward identity, Eq. (A5). Dashed lines denote path-ordered exponentials.

In addition, it makes normalization properties of valence quark distributions explicit.

In our special kinematics vectors $n_{\mu}$ and $q_{\mu}$ are proportional:

$$
n_{\mu}=\frac{n \cdot x}{q \cdot x} q_{\mu}
$$

Replacing $\gamma_{\mu} n^{\mu}$ in the definition (32) of $\hat{O}^{i}$ by $\frac{n \cdot x}{q \cdot x} \gamma_{\mu} q^{\mu}$ and integrating by parts over $d^{4} y$ in (31) we get

$$
\begin{aligned}
\Pi^{i}= & -i \int d^{4} x \exp (i p \cdot x) \int d^{4} y \exp (i q \cdot y) \frac{n \cdot x}{q \cdot x} \\
& \times \frac{\partial}{\partial y_{\mu}}\left\langle\Omega\left|T\left\{\eta(x) \bar{\Psi}^{i}\left(y+\frac{\Delta}{2}\right) \gamma_{\mu}\left[y+\frac{\Delta}{2} ; y-\frac{\Delta}{2}\right] \Psi^{i}\left(y-\frac{\Delta}{2}\right) \bar{\eta}(0)\right\}\right| \Omega\right\rangle+(\Delta \rightarrow-\Delta)
\end{aligned}
$$

The next step is to evaluate the derivative $\partial / \partial y_{\mu}$ explicitly. One gets

$$
\begin{aligned}
\frac{\partial}{\partial y_{\mu}} \bar{\Psi}^{i}\left(y+\frac{\Delta}{2}\right) \gamma_{\mu}\left[y+\frac{\Delta}{2} ; y-\frac{\Delta}{2}\right] \Psi^{i}\left(y-\frac{\Delta}{2}\right) \\
=\hat{D} \bar{\Psi}^{i}\left(y+\frac{\Delta}{2}\right)\left[y+\frac{\Delta}{2} ; y-\frac{\Delta}{2}\right] \Psi^{i}\left(y-\frac{\Delta}{2}\right)+\bar{\Psi}^{i}\left(y+\frac{\Delta}{2}\right)\left[y+\frac{\Delta}{2} ; y-\frac{\Delta}{2}\right] \hat{D} \Psi^{i}\left(y-\frac{\Delta}{2}\right) \\
\quad-\frac{i g}{2} \int_{-1}^{1} d \xi \bar{\Psi}^{i}\left(y+\frac{\Delta}{2}\right)\left[y+\frac{\Delta}{2} ; y+\xi \frac{\Delta}{2}\right] \gamma^{\alpha} \Delta^{\beta} G_{\alpha \beta}\left(y+\xi \frac{\Delta}{2}\right)\left[y+\xi \frac{\Delta}{2} ; y-\frac{\Delta}{2}\right] \Psi\left(y-\frac{\Delta}{2}\right),
\end{aligned}
$$

where $G_{\alpha \beta}$ is the gluon field strength.

Inserting the right-hand side of (A3) into (A2) we note that terms containing $\hat{D} \bar{\Psi}$ and $\hat{D} \Psi$ lead to contact terms. To this end it is convenient to have in mind the functional integral representation of the correlation function in (A2), and use the identity

$$
\exp \left(i \int \mathcal{L} d^{4} x\right) \hat{D} \Psi(y)=-\frac{\delta}{\delta \bar{\Psi}(y)} \exp \left(i \int \mathcal{L} d^{4} x\right)
$$

where $\mathcal{L}$ is the QCD Lagrangian. Making an integration by parts in the functional integral we obtain the $\delta$ function $\delta\left(x-y+\frac{\Delta}{2}\right)$ which allows to perform the integration over variable $y$ explicitly. The net result can be written as a Ward identity: 


$$
\begin{aligned}
\Pi^{i}= & \frac{1}{2} \int d^{4} x \exp (i p \cdot x) \int d y \exp (i q \cdot y) \frac{n \cdot x}{q \cdot x} \int_{-1}^{1} d \xi\langle\Omega| T\left\{\eta(x) \bar{\Psi}^{i}\left(y+\frac{\Delta}{2}\right)\right. \\
& \left.\times\left[y+\frac{\Delta}{2} ; y+\xi \frac{\Delta}{2}\right] g \gamma^{\alpha} \Delta^{\beta} G_{\alpha \beta}\left(y+\xi \frac{\Delta}{2}\right)\left[y+\xi \frac{\Delta}{2} ; y-\frac{\Delta}{2}\right] \Psi^{i}\left(y-\frac{\Delta}{2}\right) \bar{\eta}(0)\right\}|\Omega\rangle \\
& +N^{i} i \int d^{4} x \exp (i p \cdot x) \frac{n \cdot x}{q \cdot x}\left[\exp (i q \cdot x)\left\langle\Omega\left|T\left(\eta^{i}(x ;-\Delta) \bar{\eta}(0)\right)\right| \Omega\right\rangle\right. \\
& \left.-\left\langle\Omega\left|T\left(\eta(x) \bar{\eta}^{i}(0 ; \Delta)\right)\right| \Omega\right\rangle\right]+(\Delta \rightarrow-\Delta)
\end{aligned}
$$

where $N^{i}=1$ and 2 for $d$ and $u$ quarks, respectively. The nonlocal currents $\eta^{i}(x ;-\Delta)$ are defined as

$$
\begin{aligned}
& \eta^{d}(x ;-\Delta)=\epsilon^{a b c} u^{a}(x)^{T} C \gamma_{\mu} u^{b}(x) \gamma_{5} \gamma^{\mu}[x ; x-\Delta]^{c f} d^{f}(x-\Delta), \\
& \eta^{u}(x ;-\Delta)=\epsilon^{a b c} u^{f T}(x-\Delta) C \gamma_{\mu}[x-\Delta ; x]^{f a} u^{b}(x) \gamma_{5} \gamma^{\mu} d^{c}(x)
\end{aligned}
$$

The outcome of Eq. (A5) is that the complicated correlation function in (31) is written as a sum of several simpler terms. The last two terms contain one integration less compared to the original expression, and therefore they cannot generate bilocal power corrections. The first term contains explicitly a gluon field. Thus, the OPE for this term starts with higher orders in the coupling and (or) the dimension of the corresponding operators. By an explicit comparison of the OPE applied to the correlation function (31) and to its equivalent form in (A5) one can make sure that several important BPC's are transformed in this way to the LPC's related to vacuum expectation values of local operators, see Fig. 13 for the illustration. In fact, the last two terms in (A5) presumably collect all BPC's which can be reduced to vacuum condensates by low-energy theorems. Experience of practical calculations in the sum rules shows that these terms are typically the most important ones numerically. In this paper we do not take into account additional BPC's from the expansion of the term with an extra gluon in (A5).

In the limit $\Delta \rightarrow 0$ the last term disappears and we are left with the correlation function

$$
\Pi^{i}=N^{i} i \int d^{4} x \exp [i p \cdot x] \frac{n \cdot x}{q \cdot x}[\exp (i q \cdot x)-1]\langle\Omega|T(\eta(x) \bar{\eta}(0))| \Omega\rangle .
$$

Equation (A7) can further be simplified writing

$$
[\exp (i q \cdot x)-1]=i q \cdot x \int_{0}^{1} d v \exp (i v q \cdot x)
$$

so that we finally obtain

$$
\begin{aligned}
\Pi^{i} & =N^{i} \int_{0}^{1} d v n_{\mu} \frac{\partial}{\partial \tilde{p}_{\mu}} i \int d^{4} x \exp (i \tilde{p} \cdot x)\langle\Omega|T(\eta(x) \bar{\eta}(0))| \Omega\rangle \\
& =N^{i} \int_{0}^{1} d v n_{\mu} \frac{\partial}{\partial \tilde{p}_{\mu}} \Pi^{(2)}(\tilde{p})
\end{aligned}
$$

where $\tilde{p}=p+v q$, arriving at the equation in (35).

As an illustration of the use of the identity in (A5) in practical calculation, let us consider the leading perturbative contribution (PT) to the correlation function (34) for $u$ quarks, shown in Fig. 6(a). First we note that owing to the explicit presence of the coupling in the gluon field, the first term in (A5) can to this accuracy be neglected. Since

$$
\left\langle\Omega\left|T\left(\eta^{u}(x ;-\Delta) \bar{\eta}(0)\right)\right| \Omega\right\rangle_{P T}=\left\langle\Omega\left|T\left(\eta(x) \bar{\eta}^{u}(0 ; \Delta)\right)\right| \Omega\right\rangle_{P T}
$$

the remaining contribution can be rewritten as:

$$
\Pi_{\mathrm{PT}}^{u}=N^{u} i \int d^{4} x \exp (i p \cdot x) \frac{n \cdot x}{q \cdot x}[\exp (i q \cdot x)-1]\left\langle\Omega\left|T\left(\eta^{u}(x ;-\Delta) \bar{\eta}(0)\right)\right| \Omega\right\rangle_{P T} .
$$

and further using (A7), as

$$
\Pi_{P T}^{u}=N^{u} i \int_{0}^{1} d v \int d^{4} x \exp (i \tilde{p} \cdot x)(n \cdot x)\left\langle\Omega\left|T\left(\eta^{u}(x ;-\Delta) \bar{\eta}(0)\right)\right| \Omega\right\rangle_{P T}
$$

A straightforward calculation of (A12) using dimensional regularization produces 


$$
\frac{1}{4} \operatorname{Tr} \not h \Pi^{u}=\frac{1}{4 \pi^{4}}(p \cdot n)^{2} \int_{0}^{1} d v \frac{\Gamma(3-d)}{\left[-\bar{v} p_{1}^{2}-v p_{2}^{2}\right]^{3-d}} \int_{0}^{1} d u\left(9 u \bar{u}^{2}+\bar{u}^{3}\right) \cos u z
$$

Comparing with equation (34) and performing the Borel transformation according to (42) we finally arrive at the $u$-quark coefficient function in Eq. (47).

[1] J.C. Collins, D.E. Soper, and G. Sterman, in Perturbative Quantum Chromodynamics, edited by A.H. Mueller (World Scientific, Singapore, 1989), p. 1.

[2] V.N. Gribov, B.L. Ioffe, and I.Ya. Pomeranchuk, Yad. Fiz. 2, 768 (1965); B.L. Ioffe, JETP Lett. 9, 163 (1969); 10, 143 (1969); Phys. Lett. 30B, 123 (1969); V. Del Duca, S.J. Brodsky, and P. Hoyer, Phys. Rev. D 46, 931 (1992), and references therein; For a pedagogical discussion see B.L. Ioffe, V.A. Khoze, and L.N. Lipatov, Hard Processes (North-Holland, Amsterdam, 1984), Chap. 3.

[3] J. Pestieau, P. Roy, and H. Terazawa, Phys. Rev. Lett. 25, 402 (1970).

[4] M. Glück, E. Reya, and A. Vogt, Z. Phys. C 53, 127 (1992); Phys. Lett. B 306, 391 (1993).

[5] CTEQ Collaboration, J. Botts et al., Phys. Lett. B 304, 159 (1993).

[6] S. Catani, Invited talk at les Recontres de Physique de La Vallee d'Aoste, Results and Perspective in Particle Physics, La Thuile, France, 1994, Report No: DFF 207/6/94 (unpublished).

[7] E.A. Kuraev, L.N. Lipatov, and V.S. Fadin, Sov. Phys. JETP 45, 199 (1977) 199; I.I. Balitsky and L.N. Lipatov, Sov. J. Nucl. Phys. 28, 822 (1978).

[8] J.C. Collins and D.E. Soper, Nucl. Phys. B194, 445 (1982).

[9] I.I. Balitsky and V.M. Braun, Nucl. Phys. B311, 541 $(1988 / 89)$.

[10] B.L. Ioffe and V.M. Belyaev, Nucl. Phys. B310, 548
(1988)

[11] A. Manohar, Phys. Lett. B 255, 579 (1991).

[12] I.I. Balitsky and V.M. Braun, Phys. Lett. B 267, 405 (1991).

[13] I.I. Balitsky and V.M. Braun, Nucl. Phys. B361, 93 (1991).

[14] E.V. Shuryak, Rev. Mod. Phys. 65, 1 (1993).

[15] A.V. Kolesnichenko, Sov. J. Nucl. Phys. 39, 968 (1984).

[16] V.M. Belyaev and B.Yu. Blok, Phys. Lett. 167B, 99 (1986); Z. Phys. C 30, 279 (1986).

[17] B.L. Ioffe, Nucl. Phys. B188, 317 (1981); B191, 71 (1981).

[18] I.I. Balitsky, Phys. Lett. 114B, 53 (1982).

[19] B.L. Ioffe and V.M. Belyaev, Nucl. Phys. B313, 647 (1989); A.S. Gorsky et al., Z. Phys. C 44, 523 (1989); A.S. Gorsky, B.L. Ioffe, and A.Yu. Khodzhamirian, ibid. 53299 (1992); V.M. Belyaev and B.L. Ioffe, Int. J. Mod. Phys. A 6, 1533 (1991); B.L. Ioffe and A.Yu. Khodzhamirian, Report No. LMU-94-01 (hepph/9403371) (unpublished).

[20] A. Vogt, Ph.D thesis, Dortmund University, 1992.

[21] V.M. Braun, P. Gornicki, L. Mankiewicz, and A. Schafer, Phys. Lett. B 302, 291 (1993).

[22] A.P. Bakulev and A.V. Radyushkin, Phys. Lett. B 271, 233 (1991); S.V. Mikhailov and A.V. Radyushkin, Phys. Rev. D 45, 1754 (1992).

[23] A.V. Radyushkin, Phys. Lett. B 271, 218 (1991). 

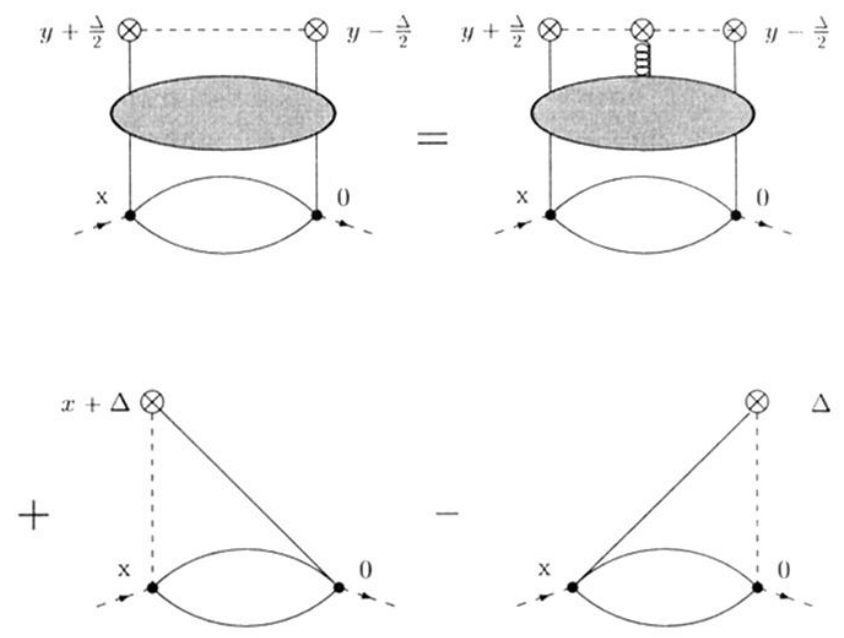

FIG. 13. Graphical illustration of the Ward identity, Eq. (A5). Dashed lines denote path-ordered exponentials. 


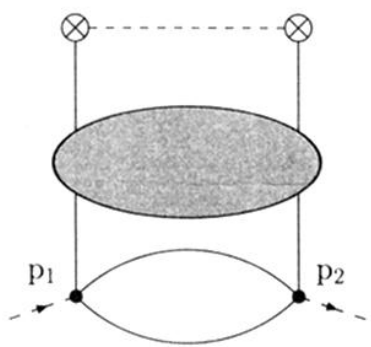

FIG. 7. Generic form of a bilocal correction in the OPE of the correlator (31). 